Research Article

\title{
Error Estimation of Euler Method for the Instationary Stokes-Biot Coupled Problem
}

\author{
Koffi Wilfrid Houédanou $\mathbb{i D}^{1}$ and Jamal Adetola ${ }^{2}$ \\ ${ }^{1}$ Faculté des Sciences et Techniques (FAST), Département de Mathématiques, Université d'Abomey-Calavi, Godomey, Benin \\ ${ }^{2}$ Universit Nationale des Sciences, Technologie, Ingnierie et Mathématiques (UNSTIM), Abomey, Benin \\ Correspondence should be addressed to Koffi Wilfrid Houédanou; khouedanou@yahoo.fr
}

Received 27 September 2021; Accepted 29 October 2021; Published 25 November 2021

Academic Editor: M. M. Bhatti

Copyright (C) 2021 Koffi Wilfrid Houédanou and Jamal Adetola. This is an open access article distributed under the Creative Commons Attribution License, which permits unrestricted use, distribution, and reproduction in any medium, provided the original work is properly cited.

\begin{abstract}
In this paper, we study a finite element computational model for solving the interaction between a fluid and a poroelastic structure that couples the Stokes equations with the Biot system. Equilibrium and kinematic conditions are imposed on the interface. A mixed Darcy formulation is employed, resulting in continuity of flux condition of essential type. A Lagrange multiplier method is used to impose weakly this condition. With the obtained finite element solutions, the error estimators are performed for the fully discrete formulations.
\end{abstract}

\section{Introduction}

The paper presents a computation friendly finite element formulation of coupled fluid motions at a free-poroelastic interface, where Navier-Stokes equations are employed for free fluid and Darcy's law is used for the permeable material. A reliable and efficient a posteriori error estimator is analysed. This is a challenging multiphysics problem that has a wide range of applications, including processes arising in gas and oil extraction from naturally or hydraulically fractured reservoirs, designing industrial filters, and blood-vessel interaction. In these applications, it is important to model properly the interaction between the free fluid with the fluid within the porous medium and to take into account the effect of the deformation of the medium. For example, geomechanical effects play an important role in hydraulic fracturing, as well as in modeling phenomena such as subsidence and compaction.

The free fluid region can be modeled by the Stokes or Navier-Stokes equations, while the flow through the deformable porous medium is modeled by the quasi-static Biot systems of poroelasticity [1]. The two regions are coupled via dynamic and kinematic interface conditions, including balance of forces, continuity of normal velocity, and a no slip or slip with friction tangential velocity condition [2-10]. These multiphysics models exhibit features of coupled Stokes-Darcy flows and fluid-structure interaction (FSI) [11-15].

The well-posedness of the mathematical model based on the Stokes-Biot system for the coupling between a fluid and a poroelastic structure is studied in [16]. A numerical study of the problem, using a Navier-Stokes equations for the fluid, is presented in $[11,17]$, utilizing a variational multiscale approach to stabilize the finite element spaces. The problem is solved using both a monolithic and a partitioned approach, with the latter requiring subiterations between the two problems.

Finite element analysis of an arbitrary Lagrangian-Eulerian method for Stokes/parabolic moving interface problem with jump coefficients has been studied in [18]. The authors in [19] studied a numerical solution of the coupled system of the time-dependent Stokes and fully dynamic Biot equations. They established stability of the scheme and derived error estimates for the fully discrete coupled scheme. Numerical errors and convergence rates for smooth problems as well as tests on realistic material parameters have 
been presented. In [20], Jing Wen and Yinnian He considered a strongly conservative discretization for the rearranged Stokes-Biot model based on the interior penalty discontinuous Galerkin method and mixed finite element method. The existence and uniqueness of solution of the numerical scheme have been presented. Then, the analysis of stability and priori error estimates has been derived. The numerical examples under uniform meshes which well validate the analysis of convergence and the strong mass conservation are presented. A staggered finite element procedure for the coupled Stokes-Biot system with fluid entry resistance has been studied by Bergkamp et al. in [21] while Ambartsumyan et al. in [22] studied flow and transport in fractured poroelastic media using Stokes flow in the fractures and the Biot model in the porous media. In Section 6 in [23], fully discrete continuous approximation has been proposed for the weak coupled mixed formulation. For the discretization of the fluid velocity and pressure, the authors have used the finite elements which include the MINI-elements, the Taylor-Hood elements, and the conforming Crouzeix-Raviart elements. For the discretization of the porous medium problem, they choose the spaces that include Raviart-Thomas and Brezzi-Douglas-Marini elements. An a priori error analysis is performed with some numerical tests confirming the convergence rates.

A posteriori error estimators are computable quantities, expressed in terms of the discrete solution and of the data that measure the actual discrete errors without the knowledge of the exact solution. They are essential to design adaptive mesh refinement algorithms which aqui-distribute the computational effort and optimize the approximation efficiency. Since the pioneering work of Babuška and Rheinboldt [24-27], adaptive finite element methods based on a posteriori error estimates have been extensively investigated.

In [28], we have proposed a family error indicator for semidiscrete approximation for the Stokes-Biot system. To the best of our knowledge, there is no a posteriori error estimation for the Stokes-Biot fluid-poroelastic structure interaction model for fully discrete finite element methods. Here, we develop such a posteriori error analysis for the fully discrete conforming finite element methods. We have got a new family of a local indicator error $\Theta_{K}$ (see equation (59) in Definition 3) and global $\Theta$ (equation (60)). The difference between this paper and our previous work [28] is that our discretization is fully discrete formulation. As an advantage, the error indicators in this work are more accessible to computation.

The schedule of the paper is as follows. Section 2 is devoted to notations and basic results that are used throughout the document. Our main results regarding a posteriori error analysis are stated in Section 3. We prove that our indicator errors are efficient, reliable, and then optimal. The global inf-sup condition is the main tool yielding the reliability. In turn, the local efficiency result is derived using the technique of bubble function introduced by R. Verfürth [29] and used in similar context by C. Carstensen [30]. Finally, this paper is summarized with further works in Section 4.

\section{Preliminaries and Notations}

2.1. Stokes-Biot Model Problem. We consider a multiphysics model problem for free fluid's interaction with a flow in a deformable porous media, where the simulation domain $\Omega \subset \mathbb{R}^{d}, d=2,3$, is a union of nonoverlapping regions $\Omega_{f}$ and $\Omega_{p}$. Here, $\Omega_{f}$ is a free fluid region with flow governed by the Stokes equations and $\Omega_{p}$ is a poroelastic material governed by the Biot system. For simplicity of notation, we assume that each region is connected. The extension to nonconnected regions is straightforward. Let $\Gamma_{f p}=\partial \Omega_{f}$ $\cap \partial \Omega_{p}$ (see Figure 1).

Let $\left(\mathbf{u}_{*}, p_{*}\right)$ be the velocity-pressure pair in $\Omega_{*}, *=f, p$, and let $\eta_{p}$ be the displacement in $\Omega_{p}$. Let $\mu>0$ be the fluid viscosity, let $\mathbf{f}_{*}$ be the body force terms, and let $q_{*}$ be external source or sink terms. Let $\mathbf{D}\left(\mathbf{u}_{f}\right)$ and $\sigma_{f}\left(\mathbf{u}_{f}, p_{f}\right)$ denote, respectively, the deformation rate tensor and the stress tensor:

$$
\begin{aligned}
\mathbf{D}\left(\mathbf{u}_{f}\right) & =\frac{1}{2}\left(\nabla \mathbf{u}_{f}+\nabla \mathbf{u}_{f}^{T}\right), \\
\sigma_{f}\left(\mathbf{u}_{f}, p_{f}\right) & =-p_{f} \mathbf{I}+2 \mu \mathbf{D}\left(\mathbf{u}_{f}\right) .
\end{aligned}
$$

In the free fluid region $\Omega_{f},\left(\mathbf{u}_{f}, p_{f}\right)$ satisfies the Stokes equations:

$$
\begin{aligned}
-\nabla \cdot \sigma_{f}\left(\mathbf{u}_{f}, p_{f}\right) & =\mathbf{f}_{f}, \quad \text { in } \Omega_{f} \times(0, T], \\
\nabla \cdot \mathbf{u}_{f} & =q_{f}, \quad \text { in } \Omega_{f} \times(0, T],
\end{aligned}
$$

where $T>0$ is the final time. Let $\sigma_{e}\left(\eta_{p}\right)$ and $\sigma_{p}\left(\eta_{p}, p_{p}\right)$ be the elastic and poroelastic stress tensors, respectively:

$$
\begin{aligned}
\sigma_{e}\left(\eta_{p}\right) & =\lambda_{p}\left(\nabla \cdot \eta_{p}\right) \mathbf{I}+2 \mu_{p} \mathbf{D}\left(\eta_{p}\right), \\
\sigma_{p}\left(\eta_{p}, p_{p}\right) & =\sigma_{e}\left(\eta_{p}\right)-\alpha p_{p} \mathbf{I},
\end{aligned}
$$

where $0<\lambda_{\min } \leq \lambda_{p}(\mathbf{x}) \leq \lambda_{\max }$ and $0<\mu_{\min } \leq \mu_{p}(\mathbf{x}) \leq \mu_{\max }$ are the Lamé parameters and $0<\alpha \leq 1$ is the Biot-Willis constant. The poroelasticity region $\Omega_{p}$ is governed by the quasistatic Biot system [23]:

$$
\begin{gathered}
-\nabla \cdot \sigma_{p}\left(\eta_{p}, p_{p}\right)=\mathbf{f}_{p}, \\
\mu K^{-1} \mathbf{u}_{p}+\nabla p_{p}=0, \quad \text { in } \Omega_{p} \times(0, T], \\
\frac{\partial}{\partial t}\left(s_{0} p_{p}+\alpha \nabla \cdot \eta_{p}\right)+\nabla \cdot \mathbf{u}_{p}=q_{p}, \quad \text { in } \Omega_{p} \times(0, T],
\end{gathered}
$$

where $s_{0} \geq 0$ is a storage coefficient and $K$ is the symmetric and uniformly positive definite rock permeability tensor, satisfying, for some constants $0<k_{\min } \leq k_{\max }$.

$$
\begin{aligned}
\forall \xi & \in \mathbb{R}^{d}, \\
k_{\min } \xi^{T} \xi & \leq \xi^{T} K(\mathbf{x}) \xi \leq k_{\max } \xi^{T} \xi, \quad \forall \mathbf{x} \in \Omega_{p} .
\end{aligned}
$$

Following [1], the interface conditions on the fluidporoelasticity interface $\Gamma_{f p}$ are mass conservation, balance of stresses, and the Beavers-Joseph-Saffman (BJS) condition [31] modeling slip with friction: 


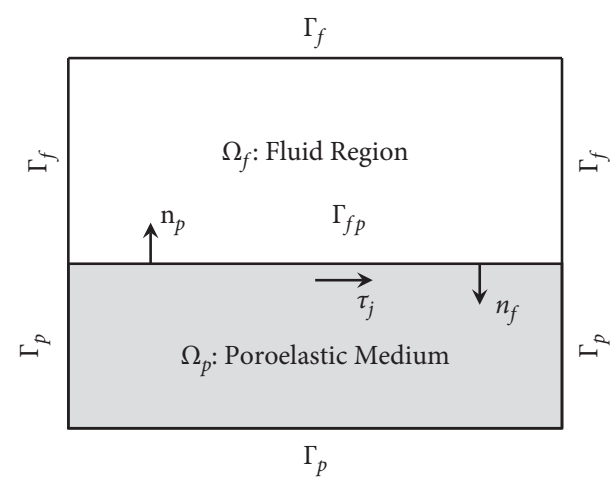

FIGURE 1: Global domain $\Omega$ consisting of the fluid region $\Omega_{f}$ and the poroelastic media region $\Omega_{p}$ separated by the interface $\Gamma_{f p}$.

$$
\begin{aligned}
& \mathbf{u}_{f} \cdot \mathbf{n}_{f}+\left(\frac{\partial \eta_{p}}{\partial t}+\mathbf{u}_{p}\right) \cdot \mathbf{n}_{p}=0, \quad \text { on } \Gamma_{f p} \times(0, T] \\
& \sigma_{f} \mathbf{n}_{f}+\sigma_{p} \mathbf{n}_{p}=0, \quad \text { on } \Gamma_{f p} \times(0, T] \\
&-\left(\sigma_{f} \mathbf{n}_{f}\right) \cdot \mathbf{n}_{f}=p_{p}, \quad \text { on } \Gamma_{f p} \times(0, T] \\
&-\left(\sigma_{f} \mathbf{n}_{f}\right) \cdot \tau_{f, j}=\mu \alpha_{\mathrm{BJF}} \sqrt{K_{j}^{-1}}\left(\mathbf{u}_{f}-\frac{\partial \eta_{p}}{\partial t}\right) \cdot \tau_{f, j} \\
& \text { on } \Gamma_{f p} \times(0, T]
\end{aligned}
$$

where $\mathbf{n}_{f}$ and $\mathbf{n}_{p}$ are the outward unit normal vectors to $\partial \Omega_{f}$ and $\partial \Omega_{p}$, respectively, $\tau_{f, j}, 1 \leq j \leq d-1$, is an orthogonal system of unit tangent vectors on $\Gamma_{f p}, K_{j}=\left(K \tau_{f, j}\right) \cdot \tau_{f, j}$, and $\alpha_{\mathrm{BJS}} \geq 0$ is an experimentally determined friction coefficient.

Here, equations (8) and (9) represent mass conservation, equation (10) is the balance of normal forces, and equation (11) is the Beavers-Joseph-Saffman conditions. We note that continuity of flux constraints the normal velocity of the solid skeleton, while the BJS condition accounts for its tangential velocity.

The above system of equations needs to be complemented by a set of boundary and initial conditions. Let $\Gamma_{f}=\partial \Omega_{f} \cap \partial \Omega$ and $\Gamma_{p}=\partial \Omega_{p} \cap \partial \Omega$. Let $\Gamma_{p}=\Gamma_{p}^{D} \cup \Gamma_{p}^{N}$. We assume that for simplicity homogeneous boundary conditions,

$$
\begin{aligned}
\mathbf{u}_{f}=0, & \text { on } \Gamma_{f} \times(0, T], \\
\eta_{p}=0, & \text { on } \Gamma_{p} \times(0, T], \\
p_{p}=0, & \text { on } \Gamma_{p}^{D} \times(0, T], \\
\mathbf{u}_{p} \cdot \mathbf{n}_{p}=0, & \text { on } \Gamma_{p}^{N} \times(0, T] .
\end{aligned}
$$

To avoid the issue with restricting the mean value of the pressure, we assume that $\left|\Gamma_{p}^{D}\right|>0$. We also assume that $\Gamma_{p}^{D}$ is not adjacent to the interface $\Gamma_{f p}$, i.e., $\operatorname{dist}\left(\Gamma_{p}^{D}, \Gamma_{f p}\right) \geq s>0$. Nonhomogeneous displacement and velocity conditions can be handled in a standard way by adding suitable extensions of the boundary data. The pressure boundary condition is natural in the mixed Darcy formulation, so nonhomogeneous pressure data would lead to an additional boundary term. We further say that the initial conditions are as follows:

$$
\begin{aligned}
& p_{p}(\mathbf{x}, 0)=p_{p, 0}(\mathbf{x}), \\
& \eta_{p}(\mathbf{x}, 0)=\eta_{p, 0}(\mathbf{x}), \quad \text { in } \Omega_{p} .
\end{aligned}
$$

Equations (2)-(10) consist of the model of the coupled Stokes and Biot flows problem that we will study below.

2.2. Weak Formulation. In this part, we first introduce some Sobolev spaces [32] and norms. If $W$ is a bounded domain of $\mathbb{R}^{d}$ and $m$ is a nonnegative integer, the Sobolev space $H^{m}(W)=W^{m, 2}(W)$ is defined in the usual way with the usual norm $\|\cdot\|_{m, W}$ and seminorm $|.|_{m, W}$. In particular, $H^{0}(W)=L^{2}(W)$, and we write $\|\cdot\|_{W}$ for $\|\cdot\|_{0, W}$. Similarly, we denote by $(\cdot, \cdot)_{W}$ the $L^{2}(W)\left[L^{2}(W)\right]^{d}$ or $\left[L^{2}(W)\right]^{d \times d}$ inner product. For shortness, if $W$ is equal to $\Omega$, we will drop the index $\Omega$, while for any $m \geq 0,\|\cdot\|_{m, *}=\|\cdot\|_{m, \Omega_{*}}$, $|\cdot|_{m, *}=|\cdot|_{m, \Omega_{*}}$, and $(., .)_{*}=(\cdot, \cdot)_{\Omega_{*}}$, for $*=f, p$. The space $H_{0}^{m}(\Omega)$ denotes the closure of $C_{0}^{\infty}(\Omega)$ in $H^{m}(\Omega)$. Let $\left[H^{m}(\Omega)\right]^{d}$ be the space of vector valued functions $\mathbf{v}=\left(v_{1}, \ldots, v_{d}\right)$ with components $v_{i}$ in $H^{m}(\Omega)$. The norm and the seminorm on $\left[H^{m}(\Omega)\right]^{d}$ are given by

$$
\begin{aligned}
\|\mathbf{v}\|_{m, \Omega} & :=\left(\sum_{i=0}^{d}\left\|v_{i}\right\|_{m, \Omega}^{2}\right)^{1 / 2}, \\
|\mathbf{v}|_{m, \Omega}: & =\left(\sum_{i=0}^{d}\left|v_{i}\right|_{m, \Omega}^{2}\right)^{1 / 2} .
\end{aligned}
$$

For a connected open subset of the boundary $E \subset \partial \Omega_{f} \cup \partial \Omega_{p}$, we write $\langle., .\rangle_{E}$ for the $L^{2}(E)$ inner product (or duality pairing); that is, for scalar valued functions $\lambda$ and $\sigma$, one defines

$$
\langle\lambda, \sigma\rangle_{E}:=\int_{E} \lambda \sigma \mathrm{d} s
$$

In the following, we derive a Lagrange multiplier type weak formulation of the system, which will be the basis for our finite element approximation. Let

$$
\begin{aligned}
& \mathbf{v}_{f}=\left\{\mathbf{v}_{f} \in H^{1}\left(\Omega_{f}\right)^{d}: \mathbf{v}_{f}=0 \text { on } \Gamma_{f}\right\}, \\
& W_{f}=L^{2}\left(\Omega_{f}\right), \\
& \mathbf{v}_{p}=\left\{\mathbf{v}_{p} \in H\left(\operatorname{div} ; \Omega_{p}\right): \mathbf{v}_{p} \cdot \mathbf{n}_{p}=0 \text { on } \Gamma_{p}^{N}\right\}, \\
& W_{p}=L^{2}\left(\Omega_{p}\right), \\
& \mathbf{X}_{p}=\left\{\xi_{p} \in H^{1}\left(\Omega_{p}\right)^{d}: \xi_{p}=0 \text { on } \Gamma_{p}\right\},
\end{aligned}
$$

where $H\left(\operatorname{div} ; \Omega_{p}\right)$ is the space of $L^{2}\left(\Omega_{p}\right)^{d}$-vectors with divergence in $L^{2}\left(\Omega_{p}\right)$ with a norm

$$
\|\mathbf{v}\|_{H\left(\operatorname{div} ; \Omega_{p}\right)}^{2}:=\|\mathbf{v}\|_{\Omega_{p}}^{2}+\|\nabla \cdot \mathbf{v}\|_{\Omega_{p}}^{2} .
$$

We define the global velocity and pressure spaces as 


$$
\begin{gathered}
\mathbf{V}=\left\{\mathbf{v}=\left(\mathbf{v}_{f}, \mathbf{v}_{p}\right) \in \mathbf{V}_{f} \times \mathbf{V}_{p}\right\}, \\
W=\left\{w=\left(w_{f}, w_{p}\right) \in W_{f} \times W_{p}\right\},
\end{gathered}
$$

with norms

$$
\begin{aligned}
& \|\mathbf{v}\|_{\mathbf{V}}^{2}:=\left\|\mathbf{v}_{f}\right\|_{\Omega_{f}}^{2}+\left\|\mathbf{v}_{p}\right\|_{H\left(\operatorname{div} ; \Omega_{p}\right)}^{2}, \\
& \|w\|_{W}^{2}=\left\|w_{f}\right\|_{\Omega_{f}}^{2}+\left\|w_{p}\right\|_{\Omega_{p}}^{2} .
\end{aligned}
$$

The weak formulation is obtained by multiplying the equations in each region by suitable test functions, integrating by parts the second-order terms in space, and utilizing the interface and boundary conditions.

Let

$$
\begin{aligned}
& a_{f}\left(\mathbf{u}_{f}, \mathbf{v}_{f}\right):=\left(2 \mu \mathbf{D}\left(\mathbf{u}_{f}\right), \mathbf{D}\left(\mathbf{v}_{f}\right)\right)_{\Omega_{f}} \\
& a_{p}^{D}\left(\mathbf{u}_{p}, \mathbf{v}_{p}\right):=\left(\mu K^{-1} \mathbf{u}_{p}, \mathbf{v}_{p}\right)_{\Omega_{p}}, \\
& a_{p}^{e}\left(\eta_{p}, \xi_{p}\right):=\left(2 \mu_{p} \mathbf{D}\left(\eta_{p}\right), \mathbf{D}\left(\xi_{p}\right)\right)_{\Omega_{p}}+\left(\lambda_{p} \nabla \cdot \eta_{p}, \nabla \cdot \xi_{p}\right)_{\Omega_{p}},
\end{aligned}
$$

be the bilinear forms related to Stokes, Darcy, and the elasticity operator, respectively. Let

$$
b_{*}(\mathbf{v}, w):=-(\nabla \cdot \mathbf{v}, w)_{\Omega_{*}} .
$$

Integration by parts in (2) and the two equations in (5) leads to the interface term as follows:

$$
I_{\Gamma_{f p}}=-\left\langle\sigma_{f} \mathbf{n}_{f}, \mathbf{v}_{f}\right\rangle_{\Gamma_{f p}}-\left\langle\sigma_{p} \mathbf{n}_{p}, \xi_{p}\right\rangle_{\Gamma_{f p}}+\left\langle p_{p}, \mathbf{v}_{p} \cdot \mathbf{n}_{p}\right\rangle_{\Gamma_{f p}} .
$$

Using the first condition for balance of normal stress in (9), we set

$$
\lambda=-\left(\sigma_{f} \mathbf{n}_{f}\right) \cdot \mathbf{n}_{f}=p_{p}, \quad \text { on } \Gamma_{f p},
$$

which will be used as a Lagrange multiplier to impose the mass conservation interface condition (8). Utilizing the BJS condition (10) and the second condition for balance of stresses in (9), we obtain

$$
I_{\Gamma_{f p}}=a_{\mathrm{BJS}}\left(\mathbf{u}_{f}, \partial_{t} \eta_{p} ; \mathbf{v}_{f}, \xi_{p}\right)+b_{\Gamma}\left(\mathbf{v}_{f}, \mathbf{v}_{p}, \xi_{p}, \lambda\right),
$$

where

$$
\begin{aligned}
a_{\mathrm{BJS}}\left(\mathbf{u}_{f}, \eta_{p} ; \mathbf{v}_{f}, \xi_{p}\right)= & \sum_{j=1}^{d-1}\left\langle\mu \alpha_{\mathrm{BJS}} \sqrt{K_{j}^{-1}}\left(\mathbf{u}_{f}-\eta_{p}\right)\right. \\
& \left.\cdot \tau_{f, j},\left(\mathbf{v}_{f}-\xi_{p}\right) \cdot \tau_{f, j}\right\rangle_{\Gamma_{f p}}, \\
b_{\Gamma}\left(\mathbf{v}_{f}, \mathbf{v}_{p}, \xi_{p} ; \mu\right)= & \left\langle\mathbf{v}_{f} \cdot \mathbf{n}_{f}+\left(\xi_{p}+\mathbf{v}_{p}\right) \cdot \mathbf{n}_{p}, \mu\right\rangle_{\Gamma_{f p}} .
\end{aligned}
$$

For the well-posedness of $b_{\Gamma}$, we require that $\lambda \in \Lambda=\left(\mathbf{V}_{p} \cdot \mathbf{n}_{p \mid \Gamma_{f p}}\right)^{\prime}$. According to the normal trace theorem, since $\mathbf{v}_{p} \in \mathbf{V}_{p} \subset H\left(\operatorname{div} ; \Omega_{p}\right)$, then $\mathbf{v}_{p} \cdot \mathbf{n}_{p} \in$ $H^{-1 / 2}\left(\partial \Omega_{p}\right)$. Furthermore, since $\mathbf{v}_{p} \cdot \mathbf{n}_{p}=0$ on $\Gamma_{p}^{N}$ and $\operatorname{dis}\left(\Gamma_{p}^{D}, \Gamma_{f p}\right) \geq s>0$, then $\mathbf{v}_{p} \cdot \mathbf{n}_{p} \in H^{-1 / 2}\left(\Gamma_{f p}\right)$, (see, e.g., [33]). Therefore, we take $\wedge=H^{\mathrm{f} / 2}\left(\Gamma_{f p}\right)$.

The Lagrange multiplier variational formulation is for $t \in(0, T]$, find $\mathbf{u}_{f}(t) \in \mathbf{V}_{f}, \quad p_{f}(t) \in W_{f}, \quad \mathbf{u}_{p}(t) \in \mathbf{V}_{p}$, $p_{p}(t) \in W_{p}, \eta_{p}(t) \in \mathbf{X}_{p}$, and $\lambda(t) \in \wedge$, such that $p_{p}(0)=p_{p, 0}$ and $\eta_{p}(0)=\eta_{p, 0}$, and for all $\mathbf{v}_{f} \in \mathbf{V}_{f}, w_{f} \in W_{f}, \mathbf{v}_{p} \in \mathbf{V}_{p}$, $w_{p} \in W_{p}, \xi_{p} \in \mathbf{X}_{p}$, and $\mu \in \wedge$,

$$
\begin{aligned}
& a_{f}\left(\mathbf{u}_{f}, \mathbf{v}_{f}\right)+a_{p}^{D}\left(\mathbf{u}_{p}, \mathbf{v}_{p}\right)+a_{p}^{e}\left(\eta_{p}, \xi_{p}\right)+a_{\mathrm{BJS}}\left(\mathbf{u}_{f}, \partial_{t} \eta_{p} ; \mathbf{v}_{f}, \xi_{p}\right) \\
& \quad+b_{f}\left(\mathbf{v}_{f}, p_{f}\right)+b_{p}\left(\mathbf{v}_{p}, p_{p}\right)+\alpha b_{p}\left(\xi_{p}, p_{p}\right)+b_{\Gamma}\left(\mathbf{v}_{f}, \mathbf{v}_{p}, \xi_{p} ; \lambda\right)=\left(\mathbf{f}_{f}, \mathbf{v}_{f}\right)_{\Omega_{f}}+\left(\mathbf{f}_{p}, \xi_{p}\right)_{\Omega_{p}}, \\
& \left(s_{0} \partial_{t} p_{p}, w_{p}\right)_{\Omega_{p}}-\alpha b_{p}\left(\partial_{t} \eta_{p}, w_{p}\right)-b_{p}\left(\mathbf{u}_{p}, w_{p}\right)-b_{f}\left(\mathbf{u}_{f}, w_{f}\right) \\
& \quad=\left(q_{f}, w_{f}\right)_{\Omega_{f}}+\left(q_{p}, w_{p}\right)_{\Omega_{p}}, \\
& b_{\Gamma}\left(\mathbf{u}_{f}, \mathbf{u}_{p}, \partial_{t} \eta_{p} ; \mu\right)=0,
\end{aligned}
$$

where we used the notation $\partial_{t}=(\partial / \partial t)$.

The assumptions on the fluid viscosity $\mu$ and the material coefficients $K, \lambda_{p}$, and $\mu_{p}$ imply that the bilinear forms $a_{f}(\cdot, \cdot), a_{p}^{D}(\cdot, \cdot)$, and $a_{p}^{e}(\cdot, \cdot)$ are coercive and continuous in the appropriate norms. In particular, there exist positive constants $c^{f}, c^{p}, c^{e}, C^{f}, C^{p}$, and $C^{e}$ such that

$$
\begin{aligned}
& c^{f}\left\|\mathbf{v}_{f}\right\|_{H^{1}\left(\Omega_{f}\right)}^{2} \leq a_{f}\left(\mathbf{v}_{f}, \mathbf{v}_{f}\right), \quad \forall \mathbf{v}_{f} \in \mathbf{V}_{f}, \\
& \quad a_{f}\left(\mathbf{v}_{f}, \mathbf{q}_{f}\right) \leq C^{f}\left\|\mathbf{v}_{f}\right\|_{H^{1}\left(\Omega_{f}\right)}\left\|\mathbf{q}_{f}\right\|_{H^{1}\left(\Omega_{f}\right)}, \quad \forall \mathbf{v}_{f}, \mathbf{q}_{f} \in \mathbf{v}_{f},
\end{aligned}
$$




$$
\begin{aligned}
& c^{p}\left\|\mathbf{v}_{p}\right\|_{L^{2}\left(\Omega_{p}\right)}^{2} \leq a_{p}^{D}\left(\mathbf{v}_{p}, \mathbf{v}_{p}\right), \quad \forall \mathbf{v}_{p} \in \mathbf{v}_{p}, \\
& a_{p}^{D}\left(\mathbf{v}_{p}, \mathbf{q}_{p}\right) \leq C^{p}\left\|\mathbf{v}_{p}\right\|_{L^{2}\left(\Omega_{p}\right)}\left\|\mathbf{q}_{p}\right\|_{L^{2}\left(\Omega_{p}\right)}, \quad \forall \mathbf{v}_{p}, \mathbf{q}_{p} \in \mathbf{V}_{p^{\prime}}, \\
& c^{e}\left\|\xi_{p}\right\|_{H^{1}\left(\Omega_{p}\right)}^{2} \leq a_{p}^{e}\left(\xi_{p}, \xi_{p}\right), \quad \forall \xi_{p} \in \mathbf{X}_{p}, \\
& a_{p}^{e}\left(\xi_{p}, \zeta_{p}\right) \leq C^{e}\left\|\xi_{p}\right\|_{H^{1}\left(\Omega_{p}\right)}\left\|\zeta_{p}\right\|_{H^{1}\left(\Omega_{p}\right)}, \quad \forall \xi_{p}, \zeta_{p} \in \mathbf{X}_{p},
\end{aligned}
$$

where (29), (30), (33), and (34) hold true thanks to Poincaré inequality and (33) and (34) also relies on Korn's inequality.

In summary, from Corollary 3.1 in [23] (p. 7), the following result holds.

Theorem 1. There exists a unique solution $\left(\mathbf{u}_{f}, p_{f}, \mathbf{u}_{p}\right.$, $\left.p_{p}, \eta_{p}, \lambda\right) \in L^{\infty}\left(0, T ; \mathbf{V}_{f}\right) \times L^{\infty}\left(0, T ; W_{f}\right) \times L^{\infty}\left(0, T ; \mathbf{V}_{p}\right) \times$ $W^{1, \infty}\left(0, T ; W_{p}\right) \times W^{1, \infty}\left(0, T ; \mathbf{X}_{p}\right) \times L^{\infty}(0, T ; \Lambda)$ to problems (26)-(28).

2.3. Finite Element Discretization. Let $\mathscr{T}_{h}^{f}$ and $\mathscr{T}_{h}^{p}$ be shaperegular and quasi-uniform partition of $\Omega_{f}$ and $\Omega_{p}$, respectively, both consisting of affine elements with maximal element diameter $h$. The two partitions may be nonmatching at the interface $\Gamma_{f p}$. For the discretization of the fluid velocity and pressure, we choose finite element spaces $\mathbf{V}_{f, h} \subset \mathbf{V}_{f}$ and $W_{f, h} \subset W_{f}$, which are assumed to be inf-sup stable. Examples of such spaces include the mini-elements, the Taylor-Hood elements, and the conforming Crouzeix-Raviart elements. For the discretization of the porous medium problem, we choose $\mathbf{V}_{p, h} \subset \mathbf{V}_{p}$ and $W_{p, h} \subset W_{p}$ to be any of well-known inf-sup stable mixed finite element spaces, such as the Raviart-Thomas or the Brezzi-Douglas-Marini spaces. The global spaces are as follows:

$$
\begin{aligned}
\mathbf{V}_{h} & :=\left\{\mathbf{v}_{h}=\left(\mathbf{v}_{f, h}, \mathbf{v}_{p, h}\right) \in \mathbf{V}_{f, h} \times \mathbf{V}_{p, h}\right\}, \\
W_{h} & =\left\{w_{h}=\left(w_{f, h}, w_{p, h}\right) \in W_{f, h} \times W_{p, h}\right\} .
\end{aligned}
$$

We employ a conforming Lagrangian finite element space $\mathbf{X}_{p, h} \subset \mathbf{X}_{p}$ to approximate the structure displacement. Note that the finite element spaces $\mathbf{V}_{f, h}, \mathbf{V}_{p, h}$, and $\mathbf{X}_{p, h}$ satisfy the prescribed homogeneous boundary conditions on the external boundaries. For the discrete Lagrange multiplier space, we take

$$
\Lambda_{h}=\mathbf{V}_{p, h} \cdot \mathbf{n}_{p \mid \Gamma_{f p}} \cdot
$$

The semidiscrete continuous-in-time problem reads that given $p_{p, h}(0)$ and $\eta_{p, h}(0)$, for $t \in(0, T]$, find $\mathbf{u}_{f, h}(t) \in \mathbf{V}_{f, h}$, $p_{f, h}(t) \in W_{f, h}, \mathbf{u}_{p, h}(t) \in \mathbf{V}_{p, h}, p_{p, h}(t) \in W_{p, h}, \eta_{p, h}(t) \in \mathbf{X}_{p, h}$, and $\lambda_{h}(t) \in \Lambda_{h}$ such that for all $\mathbf{v}_{f, h} \in \mathbf{V}_{f, h}, w_{f, h} \in W_{f, h}$, $\mathbf{v}_{p, h} \in \mathbf{V}_{p, h}, w_{p, h} \in W_{p, h}, \xi_{p, h} \in \mathbf{X}_{p, h}$, and $\mu_{h} \in \Lambda_{h}$,

$$
\begin{aligned}
& a_{f}\left(\mathbf{u}_{f, h}, \mathbf{v}_{f, h}\right)+a_{p}^{D}\left(\mathbf{u}_{p, h}, \mathbf{v}_{p, h}\right)+a_{p}^{e}\left(\eta_{p, h}, \xi_{p, h}\right) \\
& \quad+a_{\mathrm{BJS}}\left(\mathbf{u}_{f, h}, \partial_{t} \eta_{p, h}, \mathbf{v}_{f, h}, \xi_{p, h}\right)+b_{f}\left(\mathbf{v}_{f, h}, p_{f, h}\right) \\
& \quad+b_{p}\left(\mathbf{v}_{p, h}, p_{p, h}\right)+\alpha b_{p}\left(\xi_{p, h}, p_{p, h}\right)+b_{\Gamma}\left(\mathbf{v}_{f, h}, \mathbf{v}_{p, h}, \xi_{p, h} ; \lambda_{h}\right)=\left(\mathbf{f}_{f, h}, \mathbf{v}_{f, h}\right)_{\Omega_{f}}+\left(\mathbf{f}_{p, h}, \xi_{p, h}\right)_{\Omega_{p}} \\
& \left(s_{0} \partial_{t} p_{p, h}, w_{p, h}\right)_{\Omega_{p}}-\alpha b_{p}\left(\partial_{t} \eta_{p, h}, w_{p, h}\right)-b_{p}\left(\mathbf{u}_{p, h}, w_{p, h}\right)-b_{f}\left(\mathbf{u}_{f, h}, w_{f, h}\right) \\
& \quad=\left(q_{f}, w_{f, h}\right)_{\Omega_{f}}+\left(q_{p}, w_{p, h}\right)_{\Omega_{p}}, \\
& b_{\Gamma}\left(\mathbf{u}_{f, h}, \mathbf{u}_{p, h}, \partial_{t} \eta_{p, h} ; \mu_{h}\right)=0 .
\end{aligned}
$$

We will take $p_{p, h}(0)$ and $\eta_{p, h}(0)$ to be suitable projections of the initial data $p_{p, 0}$ and $\eta_{p, 0}$.

We introduce the errors for all variables as follows:

$$
\begin{aligned}
\mathbf{e}_{f} & :=\mathbf{u}_{f}-\mathbf{u}_{f, h}, \\
\mathbf{e}_{p} & :=\mathbf{u}_{p}-\mathbf{u}_{p, h}, \\
\mathbf{e}_{s} & :=\eta_{p}-\eta_{p, h}, \\
e_{f p} & :=p_{f}-p_{f, h}, \\
e_{p p} & :=p_{p}-p_{p, h} \\
e_{\lambda} & :=\lambda-\lambda_{h} .
\end{aligned}
$$

The following results hold cf. [23].
Theorem 2 (a priori error estimation). There exists a unique solution $\left(\mathbf{u}_{f, h}, p_{f, h}, \mathbf{u}_{p, h}, p_{p, h}, \eta_{p, h}, \lambda_{h}\right)$ in $L^{\infty}\left(0, T ; \mathbf{V}_{f, h}\right) \times$ $L^{\infty}\left(0, T ; W_{f, h}\right) \times L^{\infty}\left(0, T ; \mathbf{V}_{p, h}\right) \times W^{1, \infty}\left(0, T ; W_{p, h}\right) \times W^{1, \infty}$ $\left(0, T ; \mathbf{X}_{p, h}\right) \times L^{\infty}\left(0, T ; \Lambda_{h}\right)$ of the discrete problems (37)-(39), and if the solution $\left(\mathbf{u}_{f}, p_{f}, \mathbf{u}_{p}, p_{p}, \eta_{p}, \lambda\right) \in \mathbf{V}_{f} \times$ $W_{f} \times \mathbf{V}_{p} \times W_{p} \times \mathbf{X}_{p} \times \Lambda$ of the continuous problems (26)-(28) is smooth enough, then we have

$$
\begin{aligned}
\left\|\mathbf{e}_{f}\right\|_{L^{2}}\left(0, T ; H^{1}\left(\Omega_{f}\right)\right) & +\left\|\mathbf{e}_{p}\right\|_{L^{2}\left(0, T ; L^{2}\left(\Omega_{p}\right)\right)} \\
& \left.\left.+\left\|\mathbf{e}_{s}\right\|_{L^{\infty}\left(0, T ; H^{1}\right.}\left(\Omega_{p}\right)\right)+\left\|e_{p}\right\|_{L^{\infty}\left(0, T ; L^{2}\right.}\left(\Omega_{p}\right)\right) \\
& \left.+\left\|e_{f}\right\|_{L^{2}\left(0, T ; L^{2}\right.}\left(\Omega_{f}\right)\right)+\left\|e_{\lambda}\right\|_{L^{2}\left(0, T ; \Lambda_{h}\right)} \\
\leq & C\left(\mathbf{u}_{f}, p_{f}, \mathbf{u}_{p}, p_{p}, \eta_{p}, \lambda\right) h^{r}, \quad r>0 .
\end{aligned}
$$


Remark 1. For $\mathbf{W}_{h}=\left(\mathbf{v}_{f, h}, w_{f, h}, \mathbf{v}_{p, h}, w_{p, h}, \xi_{p, h}, \lambda_{h}\right) \in L^{\infty}$ $\left(0, T ; \mathbf{V}_{f, h}\right) \times L^{\infty}\left(0, T ; W_{f, h}\right) \times L^{\infty}\left(0, T ; \mathbf{V}_{p, h}\right) \times W^{1, \infty} \quad(0$, $\left.T ; W_{p, h}\right) \times W^{1, \infty}\left(0, T ; \mathbf{X}_{p, h}\right) \times L^{\infty}\left(0, T ; \Lambda_{h}\right)$, we can subtract
(37)-(39) to (26)-(28) to obtain the Galerkin orthogonality relation for all $t \in] 0, T]$ :

$$
\begin{aligned}
& a_{f}\left(\mathbf{e}_{f}(t), \mathbf{v}_{f, h}(t)\right)+a_{p}^{D}\left(\mathbf{e}_{s}(t), \xi_{p, h}(t)\right)+a_{\mathrm{BJS}}\left(\mathbf{e}_{f}(t), \partial_{t} \mathbf{e}_{s}(t) ; \mathbf{v}_{f, h}(t), \xi_{p, h}(t)\right) \\
& \quad+b_{f}\left(\mathbf{v}_{f, h}(t), e_{f}(t)\right)+b_{p}\left(\mathbf{v}_{p, h}(t), e_{p}(t)\right)+\alpha b_{p}\left(\xi_{p}(t), e_{p}(t)\right) \\
& \quad+b_{\Gamma}\left(\mathbf{v}_{f}(t), \mathbf{v}_{p}(t), \xi_{p}(t), e_{\lambda}(t)\right)+\left(s_{0} \partial_{t} e_{p}(t), w_{p, h}(t)\right) \\
& \quad-\alpha b_{p}\left(\partial_{t}(t) \mathbf{e}_{s}(t), w_{p, h}(t)\right)-b_{p}\left(\mathbf{e}_{p}(t), w_{p, h}(t)\right)-b_{f}\left(\mathbf{e}_{f}(t), w_{f, h}(t)\right)=0 .
\end{aligned}
$$

2.4. Fully Discrete Formulation. For the time discretization, we employ the backward Euler method. Let $\tau$ be the time step, $T=N \tau$, and let $t_{n}=n \tau, 0 \leq n \leq N$. Let $\delta_{\tau} u^{n}:=\left(u^{n}-\right.$ $u^{n-1} / \tau$ ) be the first-order (backward) discrete time derivation, where $u^{n}:=u\left(t_{n}\right)$. Then, the fully discrete model reads that given $p_{p, h}^{0}=p_{p, h}(0)$ and $\eta_{p, h}^{0}=\eta_{p, h}(0)$, find $\mathbf{u}_{f, h}^{n} \in \mathbf{V}_{f, h}$, $p_{f, h}^{n} \in W_{f, h}, \quad \mathbf{u}_{p, h} \in \mathbf{V}_{p, h}, \quad p_{p, h}^{n} \in W_{p, h}, \quad \eta_{p, h}^{n} \in \mathbf{X}_{p, h}$, and

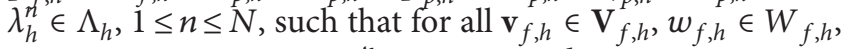
$\mathbf{v}_{p, h} \in \mathbf{V}_{p, h}, w_{p, h} \in W_{p, h}, \xi_{p, h} \in \mathbf{X}_{p, h}$, and $\mu_{h} \in \Lambda_{h}$,

$$
\begin{aligned}
& a_{f}\left(\mathbf{u}_{f, h}^{n}, \mathbf{v}_{f, h}\right)+a_{p}^{D}\left(\mathbf{u}_{p, h}^{n}, \mathbf{v}_{p, h}\right)+a_{p}^{e}\left(\eta_{p, h}^{n}, \xi_{p, h}\right) \\
& \quad+a_{\mathrm{BJS}}\left(\mathbf{u}_{f, h}^{n}, \delta_{\tau} \eta_{p, h}^{n} ; \mathbf{v}_{f, h}, \xi_{p, h}\right)+b_{f}\left(\mathbf{v}_{f, h}, p_{f, h}^{n}\right) \\
& \quad+b_{p}\left(\mathbf{v}_{p, h}, p_{p, h}^{n}\right)+\alpha b_{p}\left(\xi_{p, h}, p_{p, h}^{n}\right)+b_{\Gamma}\left(\mathbf{v}_{f, h}, \mathbf{v}_{p, h}, \xi_{p, h} ; \lambda_{h}^{n}\right)=\left(\mathbf{f}_{f}^{n}, \mathbf{v}_{f, h}\right)_{\Omega_{f}}+\left(\mathbf{f}_{p}^{n}, \xi_{p, h}\right)_{\Omega_{p}}, \\
& \left(s_{0} \delta_{\tau} p_{p, h}, w_{p, h}\right)_{\Omega_{p}}-\alpha b_{p}\left(\delta_{\tau} \eta_{p, h}^{n}, w_{p, h}\right)-b_{p}\left(\mathbf{u}_{p, h}^{n}, w_{p, h}\right)-b_{f}\left(\mathbf{u}_{f, h}^{n}, w_{f, h}\right)=\left(q_{f}^{n}, w_{f, h}\right)_{\Omega_{f}}+\left(q_{p}^{n}, w_{p, h}\right)_{\Omega_{p}}, \\
& b_{\Gamma}\left(\mathbf{u}_{f, h}^{n}, \mathbf{u}_{p, h}^{n}, \delta_{\tau} \eta_{p, h}^{n} ; \mu_{h}\right)=0 .
\end{aligned}
$$

We introduce the discrete-in-time norms as follows:

$$
\begin{aligned}
\|\phi\|_{l^{2}(0, T ; X)}^{2} & :=\left(\tau \sum_{n=0}^{N}\left\|\phi^{n}\right\|_{X}^{2}\right)^{1 / 2}, \\
\|\phi\|_{l^{\infty}(0, T ; X)}^{2} & :=\max _{0 \leq n \leq N}\left\|\phi^{n}\right\|_{X},
\end{aligned}
$$

and the errors for all variables-in-time as follows:

$$
\begin{aligned}
\mathbf{e}_{f}^{n} & :=\mathbf{u}_{f}-\mathbf{u}_{f, h}^{n}, \\
\mathbf{e}_{p}^{n} & :=\mathbf{u}_{p}-\mathbf{u}_{p, h}^{n}, \\
\mathbf{e}_{s}^{n} & :=\eta_{p}-\eta_{p, h}^{n}, \\
e_{f p}^{n} & :=p_{f}-p_{f, h}^{n}, \\
e_{p p}^{n} & :=p_{p}-p_{p, h}^{n}, \\
e_{\lambda}^{n} & :=\lambda-\lambda_{h}^{n} .
\end{aligned}
$$

Remark 2. For $n \in\{0, \ldots, N\}$ and $\mathbf{W}_{h}^{n}=\left(\mathbf{v}_{f, h}^{n}, w_{f, h}^{n}, \mathbf{v}_{p, h}^{n}\right.$, $\left.w_{p, h}^{n}, \xi_{p, h}^{n}, \mu_{h}^{n}\right) \in \mathbf{V}_{f, h} \times W_{f, h} \times \mathbf{V}_{p, h} \times W_{p, h} \times \mathbf{X}_{p, h} \times \Lambda_{h}$, we can subtract (37)-(39) to (43) and (44) to obtain the Galerkin orthogonality relation:

$$
\begin{aligned}
& a_{f}\left(\mathbf{e}_{f}^{n}, \mathbf{v}_{f, h}^{n}\right)+a_{p}^{D}\left(\mathbf{e}_{s}^{n}, \xi_{p, h}^{n}\right)+a_{\mathrm{BJS}}\left(\mathbf{e}_{f}^{n}, \delta_{\tau} \mathbf{e}_{s}^{n} ; \mathbf{v}_{f, h}^{n}, \xi_{p, h}^{n}\right) \\
& \quad+b_{f}\left(\mathbf{v}_{f, h}^{n}, e_{f}^{n}\right)+b_{p}\left(\mathbf{v}_{p, h}^{n}, e_{p}^{n}\right)+\alpha b_{p}\left(\xi_{p}^{n}, e_{p}^{n}\right) \\
& \quad+b_{\Gamma}\left(\mathbf{v}_{f}^{n}, \mathbf{v}_{p}^{n}, \xi_{p}^{n}, e_{\lambda}^{n}\right)+\left(s_{0} \delta_{\tau} e_{p}^{n}, w_{p, h}^{n}\right) \\
& \quad-\alpha b_{p}\left(\delta_{\tau} \mathbf{e}_{s}^{n}, w_{p, h}^{n}\right)-b_{p}\left(\mathbf{e}_{p}^{n}, w_{p, h}^{n}\right)-b_{f}\left(\mathbf{e}_{f}^{n}, w_{f, h}^{n}\right)=0 .
\end{aligned}
$$

\section{Error Estimation}

In order to solve the Stokes-Biot model problem by efficient adaptive finite element methods, reliable and efficient a posteriori error analysis is important to provide appropriated indicators. In this section, we first define the local 
and global indicators (Section 3.1) and then the lower and upper error bounds are derived (Sections 3.4 and 3.5).

3.1. Residual Error Estimators. The general philosophy of residual error estimators is to estimate an appropriate norm of the correct residual by terms that can be evaluated easier and that involve the data at hand. To this end, define the exact element residuals.
Definition 1 (exact element residuals). Let $n \in\{0, \ldots, N\}$ and $\quad \mathbf{W}_{h}^{n}=\left(\mathbf{v}_{f, h}^{n}, p_{f, h}^{n}, \mathbf{v}_{p, h}^{n}, w_{p, h}^{n}, \xi_{p, h}^{n}, \mu_{h}^{n}\right) \in \mathbf{V}_{f, h} \times W_{f, h} \times$ $\mathbf{V}_{p, h} \times W_{p, h} \times \mathbf{X}_{p, h} \times \Lambda_{h}$ be an arbitrary finite element function. The exact element residuals over a triangle or tetrahedra $K \in \mathscr{T}_{h}$ and over $E \in \mathscr{E}_{h}\left(\Gamma_{f p}\right)$ are defined by

$$
\begin{aligned}
\mathbf{R}_{f, K}\left(\mathbf{W}_{h}^{n}\right) & =\mathbf{f}_{f}^{n}+\nabla \cdot \sigma_{f}\left(\mathbf{v}_{f, h}^{n}, w_{f, h}^{n}\right), \\
\mathbf{R}_{p, K, 1}\left(\mathbf{W}_{h}^{n}\right) & =\mathbf{f}_{p}^{n}+\nabla \cdot \sigma_{p}\left(\xi_{p, h}^{n}, w_{p, h}^{n}\right), \\
\mathbf{R}_{p, K, 2}\left(\mathbf{W}_{h}^{n}\right) & =\mu K^{-1} \mathbf{v}_{p, h}^{n}+\nabla w_{p, h}^{n}, \\
R_{f, K}\left(\mathbf{W}_{h}^{n}\right) & =q_{f}^{n}-\nabla \cdot \mathbf{v}_{f, h}^{n}, \\
R_{p, K}\left(\mathbf{W}_{h}^{n}\right) & =q_{p}^{n}-\delta_{\tau}\left(s_{0} w_{p, h}^{n}+\alpha \nabla \cdot \xi_{p, h}^{n}\right)+\nabla \cdot \mathbf{v}_{p, h}^{n}, \\
R_{E, p f, 1}\left(\mathbf{W}_{h}^{n}\right) & =\mathbf{v}_{f, h}^{n} \cdot \mathbf{n}_{f, E}+\left(\delta_{\tau} \xi_{p}^{n}+\mathbf{v}_{p, h}^{n}\right) \cdot \mathbf{n}_{p, E}, \\
R_{E, p f, 2}\left(\mathbf{W}_{h}^{n}\right) & =w_{p, h}+\left(\sigma_{f}\left(\mathbf{v}_{f, h}^{n}, w_{f, h}^{n}\right) \mathbf{n}_{f, E}\right) \cdot \mathbf{n}_{f, E}, \\
R_{E, p f, 3}\left(\mathbf{W}_{h}^{n}\right) & =\sigma_{f}\left(\mathbf{v}_{f, h}^{n}, w_{f, h}^{n}\right) \mathbf{n}_{f, E}+\sigma_{p}\left(\xi_{p, h}^{n}, w_{p, h}^{n}\right) \mathbf{n}_{p, E}, \\
R_{E, p f, 4}\left(\mathbf{W}_{h}^{n}\right)(j) & =\left(\sigma_{f}\left(\mathbf{v}_{f, h}^{n}, w_{f, h}^{n}\right)\right) \tau_{f, E, j}+\mu \alpha_{\mathrm{BJS}} \sqrt{K_{j}^{-1}}\left(\mathbf{v}_{f, h}^{n}-\delta_{\tau} \xi_{p, h}^{n}\right) \cdot \tau_{f, E, j} .
\end{aligned}
$$

As it is common, these exact residuals are replaced by some finite-dimensional approximation called approximate element residual $\mathbf{r}_{*, K}, r_{*, K}, * \in\{f, p\}, R_{E, p f, l}, l \in\{1,2,3,4\}$. This approximation is here achieved by projecting $\mathbf{f}_{f}$ and $q_{f}$ on the space of piecewise constant functions in $\Omega_{f}$ and piecewise $\mathbb{P}^{1}$ functions in $\Omega_{p}$; more precisely for all $K \in \mathscr{T}_{h}^{f}$, we take

$$
\begin{aligned}
& \mathbf{f}_{f, K}^{n}=p_{K}\left(\mathbf{f}_{f}^{n}\right), \\
& q_{f, K}^{n}=p_{K}\left(q_{f}^{n}\right), \quad \text { with } P_{K}: L^{1}(K) \longrightarrow \mathbb{R} \text { such that } p_{K}(\phi)=\frac{1}{|K|} \int_{K} \phi(x) \mathrm{d} x .
\end{aligned}
$$

While for all $K \in \mathscr{T}_{h}^{p}$, we take $\mathbf{f}_{p, K}^{n}$ and $q_{p}^{n}$ as the unique element of $\left[\mathbb{P}^{1}(K)\right]^{d}$, respectively, $\mathbb{P}^{1}(K)$ such that

$$
\int_{K} \mathbf{f}_{p, K}^{n}(x) \cdot \mathbf{q}(x) \mathrm{d} x=\int_{K} \mathbf{f}_{p}^{n}(x) \mathbf{q}(x) \mathrm{d} x, \quad \mathbf{q} \in\left[\mathbb{P}^{1}(K)\right]^{d},
$$

respectively,

$$
\int_{K} q_{p, K}^{n}(x) q(x) \mathrm{d} x=\int_{K} q_{p}^{n}(x) q(x) \mathrm{d} x, \quad \forall q \in \mathbb{P}^{1}(K) .
$$

Thereby, we define the approximate element residuals.

Definition 2 (approximate element residuals). Let $n \in\{0 \ldots, N\} \quad$ and $\quad \mathbf{W}_{h}^{n}=\left(\mathbf{v}_{f, h}^{n}, p_{f, h}^{n}, \mathbf{v}_{p, h}^{n}, w_{p, h}^{n}, \xi_{p, h}^{n}, \mu_{h}^{n}\right) \in$ $\mathbf{V}_{f, h} \times W_{f, h} \times \mathbf{V}_{p, h} \times W_{p, h} \times \mathbf{X}_{p, h} \times \Lambda_{h}$ be an arbitrary finite element function. Then, the approximate element residuals are defined by

$$
\begin{aligned}
\mathbf{r}_{f, K}\left(\mathbf{W}_{h}^{n}\right) & =\mathbf{f}_{f, K}^{n}+\nabla \cdot \sigma_{f}\left(\mathbf{v}_{f, h}^{n}, w_{f, h}^{n}\right), \\
\mathbf{r}_{p, K, 1}\left(\mathbf{W}_{h}^{n}\right) & =\mathbf{f}_{p, K}^{n}+\nabla \cdot \sigma_{p}\left(\xi_{p, h}^{n}, w_{p, h}^{n}\right), \\
\mathbf{r}_{p, K, 2}\left(\mathbf{W}_{h}^{n}\right) & =\mu K^{-1} \mathbf{v}_{p, h}^{n}+\nabla w_{p, h}^{n}, \\
r_{f, K}\left(\mathbf{W}_{h}^{n}\right) & =q_{f, K}^{n}-\nabla \cdot \mathbf{v}_{f, h}^{n}, \\
r_{p, K}\left(\mathbf{W}_{h}^{n}\right) & =q_{p, K}^{n}-\delta_{\tau}\left(s_{0} w_{p, h}^{n}+\alpha \nabla \cdot \xi_{p, h}^{n}\right)+\nabla \cdot \mathbf{v}_{p, h}^{n} .
\end{aligned}
$$
by

Next, introduce the gradient jump in normal direction

$$
\begin{cases}\mathbf{J}_{E, \mathbf{n}_{E}, f}\left(\mathbf{U}_{h}^{n}\right):=\left[\left(2 \mu \mathbf{D}\left(\mathbf{u}_{f, h}^{n}\right)-p_{f, h}^{n} \mathbf{I}\right) \cdot \mathbf{n}_{E}\right]_{E}, & \text { if } E \in \mathscr{E}_{h}\left(\Omega_{f}\right), \\ \mathbf{J}_{E, \mathbf{n}_{E}, p}\left(\mathbf{U}_{h}^{n}\right):=\left[\left(2 \mu \mathbf{D}\left(\eta_{p, h}^{n}\right)-p_{p, h}^{n} \mathbf{I}\right) \cdot \mathbf{n}_{E}\right]_{E^{\prime}}, & \text { if } E \in \mathscr{E}_{h}\left(\Omega_{p}\right),\end{cases}
$$

where $\mathbf{I}$ is the identity matrix of $\mathbb{R}^{d \times d}$. 
Definition 3 (residual error estimators). Let $n \in\{0, \ldots, N\}$ and $\mathbf{U}_{h}^{n}=\left(\mathbf{u}_{f, h}^{n}, p_{f, h}^{n}, \mathbf{u}_{p, h}^{n}, p_{p, h}^{n}, \eta_{p, h}^{n}, \lambda_{h}^{n}\right)$ be the finite element solution of the fully discrete problems (43)-(45) in $\mathbf{V}_{f, h} \times W_{f, h} \times \mathbf{V}_{p, h} \times W_{p, h} \times \mathbf{X}_{p, h} \times \Lambda_{h}$

We define

$$
\Theta_{K}^{n}:=\left[\Theta_{K, f}^{2}\left(\mathbf{U}_{h}^{n}\right)+\Theta_{K, p}^{2}\left(\mathbf{U}_{h}^{n}\right)+\Theta_{K, p f}^{2}\left(\mathbf{U}_{h}^{n}\right)\right]^{(1 / 2)},
$$

where

$$
\begin{aligned}
\Theta_{K, f}^{2}\left(\mathbf{U}_{h}^{n}\right):= & h_{K}^{2}\left\|\mathbf{r}_{f, K}\left(\mathbf{U}_{h}^{n}\right)\right\|_{L^{2}(K)}^{2}+\left\|r_{f, K}\left(\mathbf{U}_{h}^{n}\right)\right\|_{L^{2}(K)}^{2} \\
& +\sum_{E \in \mathscr{E}_{h}\left(\Omega_{f}\right)} h_{E}\left\|\mathbf{J}_{E, \mathbf{n}_{E}, f}\left(\mathbf{U}_{h}^{n}\right)\right\|_{L^{2}(E)}^{2} \\
\Theta_{K, p}^{2}\left(\mathbf{U}_{h}^{n}\right):= & h_{K}^{2}\left(\left\|\mathbf{r}_{p, K, 1}\left(\mathbf{U}_{h}^{n}\right)\right\|_{L^{2}(K)}^{2}+\left\|\mathbf{r}_{p, K, 2}\left(\mathbf{U}_{h}^{n}\right)\right\|_{L^{2}(K)}^{2}\right) \\
& +h_{K}^{2}\left\|\operatorname{curl}\left[\mathbf{r}_{p, K, 2}\left(\mathbf{U}_{h}^{n}\right)\right]\right\|_{L^{2}(K)}^{2}+\left\|r_{p, K}\left(\mathbf{U}_{h}^{n}\right)\right\|_{L^{2}(K)}^{2} \\
& +\sum_{E \in \mathscr{E}_{h}\left(\Omega_{p}\right)} h_{E}\left\|\mathbf{J}_{E, \mathbf{n}_{E}, p}\left(\mathbf{U}_{h}^{n}\right)\right\|_{L^{2}(E)}^{2}
\end{aligned}
$$

and

$$
\begin{aligned}
\Theta_{K, p f}^{2}\left(\mathbf{U}_{h}^{n}\right):= & \sum_{E \in \mathscr{E}_{h}\left(\Gamma_{p f}\right)} h_{E}\left\|R_{E, p f, 1}\left(\mathbf{U}_{h}^{n}\right)\right\|_{L^{2}(E)}^{2} \\
& +\sum_{E \in \mathscr{C}_{h}\left(\Gamma_{p f}\right)} h_{E}\left\|R_{E, p f, 2}\left(\mathbf{U}_{h}^{n}\right)\right\|_{L^{2}(E)}^{2} \\
& +\sum_{E \in \mathscr{E}_{h}\left(\Gamma_{p f}\right)} h_{E}\left\|R_{E, p f, 3}\left(\mathbf{U}_{h}^{n}\right)\right\|_{L^{2}(E)}^{2} \\
& +\sum_{E \in \mathscr{E}_{h}\left(\Gamma_{p f}\right)} h_{E}\left\{\sum_{j=1}^{d-1}\left\|R_{E, p f, 4}\left(\mathbf{U}_{h}^{n}\right)(j)\right\|_{L^{2}(E)}^{2}\right\} .
\end{aligned}
$$

Then, the residual error estimator is locally defined by

$$
\Theta_{K}:=\min _{0 \leq n \leq N} \Theta_{K}^{n}, \quad \forall K \in \mathscr{T}_{h} .
$$

The global residual error estimator is given by

$$
\Theta:=\left(\sum_{K \in \mathscr{T}_{h}} \Theta_{K}^{2}\right)^{1 / 2} .
$$

Furthermore, denote the local approximation terms by

$$
\zeta_{K}^{n}:=\left[\zeta_{K, f}\left(\mathbf{U}_{h}^{n}\right)^{2}+\zeta_{K, p}\left(\mathbf{U}_{h}^{n}\right)^{2}\right]^{1 / 2},
$$

where

$$
\begin{aligned}
\zeta_{K, f}\left(\mathbf{U}_{h}^{n}\right)^{2}:= & h_{K}^{2}\left\|\mathbf{R}_{f, K}\left(\mathbf{U}_{h}^{n}\right)-\mathbf{r}_{f, K}\left(\mathbf{U}_{h}^{n}\right)\right\|_{L^{2}(K)}^{2} \\
& +h_{K}^{2}\left\|R_{f, K}\left(\mathbf{U}_{h}^{n}\right)-r_{f, K}\left(\mathbf{U}_{h}^{n}\right)\right\|_{L^{2}(K)}^{2}, \quad \text { if } K \in \mathscr{T}_{h}^{f}, \\
\zeta_{K, p}\left(\mathbf{U}_{h}^{n}\right)^{2}:= & h_{K}^{2}\left\|\mathbf{R}_{p, K, 1}\left(\mathbf{U}_{h}^{n}\right)-\mathbf{r}_{p, K, 1}\left(\mathbf{U}_{h}^{n}\right)\right\|_{L^{2}(K)}^{2} \\
& +h_{K}^{2}\left\|R_{p, K}\left(\mathbf{U}_{h}^{n}\right)-r_{p, K}\left(\mathbf{U}_{h}^{n}\right)\right\|_{L^{2}(K)}^{2}, \quad \text { if } K \in \mathscr{T}_{h}^{p} .
\end{aligned}
$$

We set

$$
\begin{aligned}
\zeta_{K} & :=\min _{0 \leq n \leq N} \zeta_{K}^{n}, \\
\zeta & :=\left(\sum_{K \in \mathscr{T}_{h}} \zeta_{K}^{2}\right)^{1 / 2} .
\end{aligned}
$$

Remark 3. The residual character of each term on the righthand sides of (55)-(58) is quite clear since if $\mathbf{U}_{h}$ would be the exact solution of (2)-(10), then they would vanish.

\subsection{Analytical Tools}

3.2.1. Inverse Inequalities. In order to derive the lower error bounds, we proceed similarly as in [30,34] (see also [35]), by applying inverse inequalities and the localization technique based on simplex-bubble and face-bubble functions. To this end, we recall some notation and introduce further preliminary results. Given $K \in \mathscr{T}_{h}$ and $E \in \mathscr{E}(K)$, we let $b_{K}$ and $b_{E}$ be the usual simplex-bubble and face-bubble functions (see (1.5) and (1.6) in [29]), respectively. In particular, $b_{K}$ satisfies $b_{K} \in \mathbb{P}^{3}(K), \quad \operatorname{supp}\left(b_{K}\right) \subseteq K, \quad b_{K}=0$, on $\partial K$, and $0 \leq b_{K} \leq 1$, on $K . \quad$ Similarly, $\quad b_{E} \in \mathbb{P}^{2}(K)$, $\operatorname{supp}\left(b_{E}\right) \subseteq \omega_{E}:=\left\{K^{\prime} \in \mathscr{T}_{h}: E \in \mathscr{E}\left(K^{\prime}\right)\right\}, \quad b_{E}=0$, on $\partial K \backslash E$ and $0 \leq b_{E} \leq 1$, in $\omega_{E}$. We also recall from [36] that, given $k \in \mathbb{N}$, there exists an extension operator $L: C(E) \longrightarrow C(K)$ that satisfies $L(p) \in \mathbb{P}^{k}(K)$ and $L(p)_{\mid E}=p, \forall p \in \mathbb{P}^{k}(E)$. A corresponding vectorial version of $L$, that is, the componentwise application of $L$, is denoted by $\mathbf{L}$. Additional properties of $b_{K}, b_{E}$, and $L$ are collected in the following lemma (see [36]).

Lemma 1. Given $k \in \mathbb{N}^{*}$, there exist positive constants depending only on $k$ and shape-regularity of the triangulations (minimum angle condition), such that for each simplex $K$ and $E \in \mathscr{E}(K)$, there hold

$$
\begin{array}{r}
\|\phi\|_{K} \lesssim\left\|\phi b_{K}^{1 / 2}\right\|_{K} \lesssim\|\phi\|_{K}, \quad \forall \phi \in \mathbb{P}^{k}(K), \\
\left|\phi b_{K}\right|_{1, K} \lesssim h_{K}^{-1}\|\phi\|_{K}, \quad \forall \phi \in \mathbb{P}^{k}(K), \\
\|\psi\|_{E} \lesssim\left\|b_{E}^{1 / 2} \psi\right\|_{E} \lesssim\|\psi\|_{E}, \quad \forall \psi \in \mathbb{P}^{k}(E), \\
\|L(\psi)\|_{K}+h_{E}|L(\psi)|_{1, K} \lesssim h_{E}^{1 / 2}\|\psi\|_{E}, \quad \forall \psi \in \mathbb{P}^{k}(E) .
\end{array}
$$

Lemma 2 (continuous trace inequality). There exists a positive constant $\beta_{1}>0$ depending only on $\sigma_{0}$ such that 


$$
\|\mathbf{v}\|_{\partial K}^{2} \leq \beta_{1}\|\mathbf{v}\|_{K}\|\mathbf{v}\|_{1, K}, \quad \forall K \in \mathscr{T}_{h}, \forall \mathbf{v} \in\left[H^{1}(K)\right]^{d} .
$$

3.2.2. Clément Interpolation Operator. In order to derive the upper error bounds, we introduce the Clément interpolation operator $\mathrm{I}_{\mathrm{C}}^{0}: H_{0}^{1}(\Omega) \longrightarrow \mathscr{P}_{c}^{b}\left(\mathscr{T}_{h}\right)$ that approximates optimally nonsmooth functions by continuous piecewise linear functions:

$$
\mathscr{P}_{c}^{b}\left(\mathscr{T}_{h}\right):=\left\{v \in C^{0}(\bar{\Omega}): v_{\mid K} \in \mathbb{P}^{1}(K), \forall K \in \mathscr{T}_{h} \text { and } v=0 \text {, on } \partial \Omega\right\} .
$$

In addition, we will make use of a vector valued version of $\mathrm{I}_{\mathrm{Cl}}^{0}$, that is, $\mathbf{I}_{\mathrm{Cl}}^{0}:\left[H_{0}^{1}(\Omega)\right]^{d} \longrightarrow\left[\mathscr{P}_{c}^{b}\left(\mathscr{T}_{h}\right)\right]^{d}$, which is defined component-wise by $\mathrm{I}_{\mathrm{Cl}}^{0}$. The following lemma establishes the local approximation properties of $\mathrm{I}_{\mathrm{Cl}}^{0}$ (and hence of $\mathbf{I}_{\mathrm{Cl}}^{0}$ ), for a proof (see Section 3 in [37]).

Lemma 3. There exist constants $C_{1}, C_{2}>0$, independent of $h$, such that for all $v \in H_{0}^{1}(\Omega)$, there hold

$$
\begin{array}{ll}
\left\|v-\mathrm{I}_{\mathrm{Cl}}^{0}(v)\right\|_{K} \leq C_{1} h_{K}\|v\|_{1, \Delta(K)}, & \forall K \in \mathscr{T}_{h}, 0.2 \mathrm{~cm}, \\
\left\|v-\mathrm{I}_{\mathrm{Cl}}^{0}(v)\right\|_{E} \leq C_{2} h_{E}^{1 / 2}\|v\|_{1, \Delta(E)}, & \forall E \in \mathscr{E}_{h},
\end{array}
$$

where $\Delta(K):=\cup\left\{K^{\prime} \in \mathscr{T}_{h}: K^{\prime} \cap K \neq \varnothing\right\}$ and $\Delta(E):=$ $\cup\left\{K^{\prime} \in \mathscr{T}_{h}: K^{\prime} \cap E \neq \varnothing\right\}$.

\subsubsection{Helmholtz Decomposition}

Lemma 4 (see [7]). There exists $C_{p}>0$ such that every $\mathbf{v}_{p} \in \mathbf{H}\left(\operatorname{div} ; \Omega_{p}\right)$ can be decomposed as $\mathbf{v}_{p}=\mathbf{w}+\operatorname{curl} \beta$, where $\mathbf{w} \in\left[H^{1}\left(\Omega_{p}\right)\right]^{d}, \beta \in H^{1}\left(\Omega_{p}\right), \int_{\Omega_{p}} \beta(x) d x=0$, and

$$
\|\mathbf{w}\|_{1, \Omega_{p}}+\|\beta\|_{1, \Omega_{p}} \leq C_{p}\left\|\mathbf{v}_{p}\right\|_{\mathbf{H}\left(\mathrm{div} ; \Omega_{p}\right)} .
$$

3.3. Error Equation. We set $\mathbf{H}=\mathbf{V}_{f} \times W_{f} \times \mathbf{V}_{p} \times W_{p}$ $\times \mathbf{X}_{p} \times \Lambda$ and $\mathbf{H}_{h}=\mathbf{V}_{f, h} \times W_{f, h} \times \mathbf{V}_{p, h} \times W_{p, h} \times \mathbf{X}_{p, h} \times \Lambda_{h}$.

For $n \in\{0, \ldots, N\}$, let $\mathbf{U}^{n}=\left(\mathbf{u}_{f}^{n}, p_{f}^{n}, \mathbf{u}_{p}^{n}, p_{p}^{n}, \eta_{p}^{n}, \lambda^{n}\right) \in \mathbf{H}$ and $\mathbf{W}=\left(\mathbf{v}_{f}^{n}, w_{f}^{n}, \mathbf{v}_{p}^{n}, w_{p}^{n}, \xi_{p}^{n}, \mu^{n}\right) \in \mathbf{H}$. We define now the operator $\mathbf{A}$ by

$$
\begin{aligned}
\mathbf{A}\left(\mathbf{U}^{n}, \mathbf{W}^{n}\right):= & a_{f}\left(\mathbf{u}_{f}^{n}, \mathbf{v}_{f}^{n}\right)+a_{p}^{D}\left(\mathbf{u}_{p}^{n}, \mathbf{v}_{p}^{n}\right)+b_{p}\left(\mathbf{v}_{p}^{n}, p_{p}^{n}\right)+\alpha b_{p}\left(\xi_{p}^{n}, p_{p}^{n}\right) \\
& +b_{\Gamma}\left(\mathbf{v}_{f}^{n}, \mathbf{v}_{p}^{n}, \xi_{p}^{n}, \lambda^{n}\right)+\left(s_{0} \delta_{\tau} p_{p}^{n}, w_{p}^{n}\right)_{\Omega_{p}}-\alpha b_{p}\left(\delta_{\tau} \eta_{p}^{n}, w_{p}^{n}\right) \\
& -b_{p}\left(\mathbf{u}_{p}^{n}, w_{p}^{n}\right)-b_{f}\left(\mathbf{u}_{f}^{n}, w_{f}^{n}\right)+b_{\Gamma}\left(\mathbf{u}_{f}^{n}, \mathbf{u}_{p}^{n}, \delta_{\tau} \eta_{p}^{n} ; \mu^{n}\right), \\
\mathbf{F}\left(\mathbf{W}^{n}\right):= & \left(\mathbf{f}_{f}^{n}, \mathbf{v}_{f}^{n}\right)_{\Omega_{f}}+\left(\mathbf{f}_{p}^{n}, \xi_{p}^{n}\right)+\left(q_{f}^{n}, w_{f}^{n}\right)_{\Omega_{f}}+\left(q_{p}^{n}, w_{p}^{n}\right)_{\Omega_{p}}
\end{aligned}
$$

Then, the continuous problems (26)-(28) are equivalent to the following: for $n \in\{0, \ldots, N\}$, find $\mathbf{U}^{n} \in \mathbf{H}$ such that we have

$$
\mathbf{A}\left(\mathbf{U}^{n}, \mathbf{W}^{n}\right)=\mathbf{F}\left(\mathbf{W}^{n}\right), \quad \forall \mathbf{W}^{n} \in \mathbf{H} .
$$

We define the discrete version by the same way: find $\mathbf{U}_{h}^{n} \in \mathbf{H}_{h}$ such that 


$$
\mathbf{A}\left(\mathbf{U}_{h}^{n}, \mathbf{W}_{h}^{n}\right)=\mathbf{F}\left(\mathbf{W}_{h}^{n}\right), \quad \forall \mathbf{W}_{h}^{n} \in \mathbf{H}_{h}
$$

Since for all $n \in\{0, \ldots, N\}$ and $\mathbf{W}_{h}^{n} \in \mathbf{H}_{h}, \mathbf{A}\left(\mathbf{U}^{n}-\right.$ $\left.\mathbf{U}_{h}^{n}, \mathbf{W}_{h}^{n}\right)=0$, then from (70), we obtain

$$
\begin{aligned}
\mathbf{A}\left(\mathbf{U}^{n}-\mathbf{U}_{h}^{n}, \mathbf{W}^{n}\right)= & \mathbf{A}\left(\mathbf{U}^{n}-\mathbf{U}_{h}^{n}, \mathbf{W}^{n}-\mathbf{W}_{h}^{n}\right) \\
= & \mathbf{A}\left(\mathbf{U}^{n}, \mathbf{W}^{n}-\mathbf{W}_{h}^{n}\right)-\mathbf{A}\left(\mathbf{U}_{h}^{n}, \mathbf{W}^{n}-\mathbf{W}_{h}^{n}\right) \\
= & \mathbf{F}\left(\mathbf{W}^{n}-\mathbf{W}_{h}^{n}\right)-\mathbf{A}\left(\mathbf{U}_{h}^{n}, \mathbf{W}^{n}-\mathbf{W}_{h}^{n}\right) \\
= & \left(\mathbf{f}_{f}^{n}, \mathbf{v}_{f}^{n}-\mathbf{v}_{f, h}^{n}\right)_{\Omega_{f}}+\left(\mathbf{f}_{p}^{n}, \xi_{p}^{n}-\xi_{p, h}^{n}\right)_{\Omega_{p}} \\
& +\left(q_{f}^{n}, w_{f}^{n}-w_{f, h}^{n}\right)_{\Omega_{f}}+\left(q_{p}^{n}, w_{p}^{n}-w_{p, h}^{n}\right)_{\Omega_{p}}-\mathbf{A}\left(\mathbf{U}_{h}^{n}, \mathbf{W}^{n}-\mathbf{W}_{h}^{n}\right) \\
= & \left(\mathbf{f}_{f}^{n}-\mathbf{f}_{f, h}^{n}, \mathbf{v}_{f}^{n}-\mathbf{v}_{f, h}^{n}\right)_{\Omega_{f}}+\left(\mathbf{f}_{p}^{n}-\mathbf{f}_{p, h}^{n}, \xi_{p}^{n}-\xi_{p, h}^{n}\right)_{\Omega_{p}} \\
& +\left(q_{f}^{n}-q_{f, h}^{n}, w_{f}^{n}-w_{f, h}^{n}\right)_{\Omega_{f}}+\left(q_{p}^{n}-q_{p, h}^{n}, w_{p}^{n}-w_{p, h}^{n}\right)_{\Omega_{p}} \\
& +\left(\mathbf{f}_{f, h}^{n}, \mathbf{v}_{f}^{n}-\mathbf{v}_{f, h}^{n}\right)_{\Omega_{f}}+\left(\mathbf{f}_{p, h}^{n}, \xi_{p}^{n}-\xi_{p, h}^{n}\right)_{\Omega_{p}}+\left(q_{f, h}^{n}, w_{f}^{n}-w_{f, h}^{n}\right)_{\Omega_{f}}+\left(q_{p, h}^{n}, w_{p}^{n}-w_{p, h}^{n}\right)_{\Omega_{p}} \\
& -\mathbf{A}\left(\mathbf{U}_{h}^{n}, \mathbf{W}^{n}-\mathbf{W}_{h}^{n}\right) .
\end{aligned}
$$

Now, we set

Then, from (72), we can write

$$
\mathbf{A}\left(\mathbf{U}^{n}-\mathbf{U}_{h}^{n}, \mathbf{W}^{n}\right)=\sum_{K \in \mathscr{T}_{h}} \mathbf{A}_{K}\left(\mathbf{U}^{n}-\mathbf{U}_{h}^{n}, \mathbf{W}^{n}\right) .
$$

$$
\begin{aligned}
\mathbf{A}_{K}\left(\mathbf{U}^{n}-\mathbf{U}_{h}^{n}, \mathbf{W}^{n}\right):= & \left(\mathbf{f}_{f}^{n}-\mathbf{f}_{f, h}^{n}, \mathbf{v}_{f}^{n}-\mathbf{v}_{f, h}^{n}\right)_{\Omega_{f} \cap K}+\left(\mathbf{f}_{p}^{n}-\mathbf{f}_{p, h}^{n}, \xi_{p}^{n}-\xi_{p, h}^{n}\right)_{\Omega_{p} \cap K} \\
& +\left(q_{f}^{n}-q_{f, h}^{n}, w_{f}^{n}-w_{f, h}^{n}\right)_{\Omega_{f} \cap K}+\left(q_{p}^{n}-q_{p, h}^{n}, w_{p}^{n}-w_{p, h}^{n}\right)_{\Omega_{p} \cap K} \\
& +\left(\mathbf{f}_{f, h}^{n}, \mathbf{v}_{f}^{n}-\mathbf{v}_{f, h}^{n}\right)_{\Omega_{f} \cap K}+\left(\mathbf{f}_{p, h}^{n}, \xi_{p}^{n}-\xi_{p, h}^{n}\right)_{\Omega_{p} \cap K} \\
& +\left(q_{f, h}^{n}, w_{f}^{n}-w_{f, h}^{n}\right)_{\Omega_{f} \cap K}+\left(q_{p, h}^{n}, w_{p}^{n}-w_{p, h}^{n}\right)_{\Omega_{p} \cap K}-\mathbf{A}_{K}\left(\mathbf{U}_{h}^{n}, \mathbf{W}^{n}-\mathbf{W}_{h}^{n}\right) \\
= & \left(\mathbf{R}_{f, K}\left(\mathbf{U}_{h}^{n}\right)-\mathbf{r}_{f, K}\left(\mathbf{U}_{h}^{n}\right), \mathbf{v}_{f}^{n}-\mathbf{v}_{f, h}^{n}\right)_{\Omega_{f} \cap K}+\left(R_{f, K}\left(\mathbf{U}_{h}^{n}\right)-r_{f, K}\left(\mathbf{U}_{h}^{n}\right), w_{f}^{n}-w_{f, h}^{n}\right)_{\Omega_{f} \cap K} \\
& +\left(\mathbf{R}_{p, K, 1}\left(\mathbf{U}_{h}^{n}\right)-\mathbf{r}_{p, K, 1}\left(\mathbf{U}_{h}^{n}\right), \xi_{p}^{n}-\xi_{p, h}^{n}\right)_{\Omega_{p} \cap K} \\
& +\left(R_{p, K}\left(\mathbf{U}_{h}^{n}\right)-r_{p, K}\left(\mathbf{U}_{h}^{n}\right), w_{p}^{n}-w_{p, h}^{n}\right)_{\Omega_{p} \cap K}+\mathbf{B}_{K}\left(\mathbf{U}_{h}^{n}, \mathbf{W}^{n}-\mathbf{W}_{h}^{n}\right),
\end{aligned}
$$

where

$$
\begin{aligned}
\mathbf{B}_{K}\left(\mathbf{U}_{h}^{n}, \mathbf{W}^{n}-\mathbf{W}_{h}^{n}\right)= & \left(\mathbf{f}_{f, K}^{n}, \mathbf{v}_{f}^{n}-\mathbf{v}_{f, h}^{n}\right)_{\Omega_{f} \cap K}+\left(\mathbf{f}_{p, K}^{n}, \xi_{p}^{n}-\xi_{p, h}^{n}\right)_{\Omega_{p} \cap K} \\
& +\left(q_{f, K}^{n}, w_{f}^{n}-w_{f, h}^{n}\right)_{\Omega_{f} \cap K}+\left(q_{p, K}^{n}, w_{p}^{n}-w_{p, h}^{n}\right)_{\Omega_{p} \cap K}-\mathbf{A}_{K}\left(\mathbf{U}_{h}^{n}, \mathbf{W}^{n}-\mathbf{W}_{h}^{n}\right) .
\end{aligned}
$$

3.4. Reliability of the A Posteriori Error Estimator. The first main result is given by the following theorem.
Theorem 3 (upper error bound). Let $\mathbf{U}=\left(\mathbf{U}^{n}\right)_{n=0}^{N}$ such that $\mathbf{U}^{n}=\left(\mathbf{u}_{f}^{n}, p_{f}^{n}, \mathbf{u}_{p}^{n}, p_{p}^{n}, \eta_{p}^{n}, \lambda^{n}\right) \in \mathbf{H}$ be the exact solution and 
$\mathbf{U}_{h}=\left(\mathbf{U}_{h}^{n}\right)_{n=0}^{N}$ with $\mathbf{U}_{h}^{n}=\left(\mathbf{u}_{f, h}^{n}, p_{f, h}^{n}, \mathbf{u}_{p, h}^{n}, p_{p, h}^{n}, \eta_{p, h}^{n}, \lambda_{h}^{n}\right) \in \mathbf{H}_{h}$ be the finite element solution. Then, there exist a positive constant $C_{\text {upper }}$ such that the error is bounded globally from above by

$$
\left\|\mathbf{U}-\mathbf{U}_{h}\right\|_{l^{\infty}\left(0, T ; \mathbf{H}_{h}\right)} \leq C_{\text {upper }}(\Theta+\zeta)
$$

Proof. Let $n \in\{0, \ldots, N\}$. We consider the residual equation (73), and we set $\mathbf{W}^{n}=\left(\mathbf{v}_{f}^{n}, w_{f}^{n}, \mathbf{v}_{p}^{n}, w_{p}^{n}, \xi_{p}^{n}, \mu^{n}\right)$. We take
$\mathbf{W}_{h}^{n}=\left(\mathbf{v}_{f, h}^{n}, 0, \mathbf{v}_{p, h}^{n}, 0, \xi_{p, h}^{n}, 0\right) \quad$ with $\quad \mathbf{v}_{f, h}^{n}=\mathbf{I}_{\mathrm{Cl}}^{0}\left(\mathbf{v}_{f}^{n}\right) \quad$ and $\xi_{p, h}^{n}=\mathbf{I}_{\mathrm{Cl}}^{0}\left(\xi_{p}^{n}\right)$. As $\mathbf{v}_{p}^{n} \in \mathbf{H}\left(\operatorname{div} ; \Omega_{p}\right)$, then by Lemma $4, \mathbf{v}_{p}^{n}$ admits the decomposition $\mathbf{v}_{p}^{n}=\mathbf{w}_{p}^{n}+\operatorname{curl} \beta^{n}$ where $\mathbf{w}_{p}^{n} \in\left[H^{1}\left(\Omega_{p}\right)\right]^{d}$ and $\beta^{n} \in H^{1}\left(\Omega_{p}\right)$ with $\int_{\Omega_{p}} \beta^{n}(x) \mathrm{d} x=0$ and $\left\|\mathbf{w}_{p}^{n}\right\|_{\left[H^{1}\left(\Omega_{p}\right)\right]^{d}}+\left\|\beta^{n}\right\|_{H^{1}\left(\Omega_{p}\right)} \leq C_{p}^{n}\left\|\mathbf{v}_{p}^{n}\right\|_{\mathbf{V}_{p}}$. We consider $\mathbf{v}_{p, h}^{n}=\mathbf{w}_{p, h}^{n}+\operatorname{curl} \beta_{p, h}^{n}$ with $\mathbf{w}_{p, h}^{n}=\mathbf{I}_{\mathrm{Cl}}\left(\mathbf{w}_{p}^{n}\right)$ and $\beta_{p, h}^{n}=$ $I_{\mathrm{Cl}}\left(\beta_{p}^{n}\right)$. Thus, $\mathbf{v}_{p}^{n}-\mathbf{v}_{p, h}^{n}=\left(\mathbf{w}_{p}^{n^{n}}-\mathbf{w}_{p, h}^{n}\right)+\operatorname{curl}\left(\beta_{p}^{n}-\beta_{p, h}^{n}\right)$. Now, we recall

$$
\begin{aligned}
\mathbf{B}_{K}\left(\mathbf{U}_{h}^{n}, \mathbf{W}^{n}-\mathbf{W}_{h}^{n}\right)= & \left(\mathbf{f}_{f, K}^{n}, \mathbf{v}_{f}^{n}-\mathbf{v}_{f, h}^{n}\right)_{\Omega_{f} \cap K}+\left(\mathbf{f}_{p, K}^{n}, \xi_{p}^{n}-\xi_{p, h}^{n}\right)_{\Omega_{p} \cap K} \\
& +\left(q_{f, K}^{n}, w_{f}^{n}-w_{f, h}^{n}\right)_{\Omega_{f} \cap K}+\left(q_{p, K}^{n}, w_{p}^{n}-w_{p, h}^{n}\right)_{\Omega_{p} \cap K}-\mathbf{A}_{K}\left(\mathbf{U}_{h}^{n}, \mathbf{W}^{n}-\mathbf{W}_{h}^{n}\right) .
\end{aligned}
$$

Therefore, integrating by parts element by element, we may write

$$
\mathbf{B}_{K}\left(\mathbf{U}_{h}^{n}, \mathbf{W}^{n}-\mathbf{W}_{h}^{n}\right)=\sum_{i=1}^{12} \Upsilon_{i, K}
$$

where

$$
\begin{aligned}
\Upsilon_{1, K}:= & \left(\mathbf{r}_{f, K}\left(\mathbf{U}_{h}^{n}\right), \mathbf{v}_{f}^{n}-\mathbf{v}_{f, h}^{n}\right)_{\Omega_{f} \cap K^{\prime}} \\
\Upsilon_{2, K}:= & \left(\mathbf{r}_{p, K, 1}\left(\mathbf{U}_{h}^{n}\right), \xi_{p}^{n}-\xi_{p, h}^{n}\right)_{\Omega_{p} \cap K^{\prime}} \\
\Upsilon_{3, K}:= & -\left(\mathbf{r}_{p, K, 2}\left(\mathbf{U}_{h}^{n}\right), \mathbf{w}_{p}^{n}-\mathbf{w}_{p, h}^{n}\right)_{\Omega_{p} \cap K^{\prime}} \\
\Upsilon_{4, K}:= & -\left(\operatorname{curl} \mathbf{r}_{p, K, 2}\left(\mathbf{U}_{h}^{n}\right), \beta_{p}^{n}-\beta_{p, h}^{n}\right)_{\Omega_{p} \cap K^{\prime}} \\
\Upsilon_{5, K}:= & \left(r_{f, K}\left(\mathbf{U}_{h}^{n}\right), w_{f}^{n}\right)_{\Omega_{f} \cap K^{\prime}} \\
\Upsilon_{6, K}:= & \left(r_{p, K}\left(\mathbf{U}_{h}^{n}\right), w_{p}^{n}\right)_{\Omega_{p} \cap K^{\prime}} \\
\Upsilon_{7, K}:= & -\sum_{E \in \mathscr{O}_{h}\left(\Omega_{f} \cap K\right)}\left(\mathbf{J}_{E, \mathbf{n}_{E}, f}\left(\mathbf{U}_{h}^{n}\right), \mathbf{v}_{f}^{n}-\mathbf{v}_{f, h}^{n}\right)_{E^{\prime}} \\
\Upsilon_{8, K}:= & -\sum_{E \in \mathscr{E}_{h}\left(\Omega_{p} \cap K\right)}\left(\mathbf{J}_{E, \mathbf{n}_{E}, p}\left(\mathbf{U}_{h}^{n}\right), \xi_{p}^{n}-\xi_{p, h}^{n}\right)_{E}, \\
\Upsilon_{9, K}:= & \sum_{E \in \mathscr{C}_{h}\left(\Gamma_{f p}\right)}\left(R_{E, p f, 1}\left(\mathbf{U}_{h}^{n}\right),\left(\mathbf{v}_{f}^{n}-\mathbf{v}_{f, h}^{n}\right) \cdot \mathbf{n}_{f, E}\right)_{E}, \\
\Upsilon_{10, K}:= & \sum_{E \in \mathscr{E}_{h}\left(\Gamma_{f p}\right)}\left(R_{E, p f, 2}\left(\mathbf{U}_{h}^{n}\right),\left(\mathbf{v}_{f}^{n}-\mathbf{v}_{f, h}^{n}\right) \cdot \mathbf{n}_{f, E}\right)_{E}, \\
\Upsilon_{11, K}:= & \sum_{E \in \mathscr{E}_{h}\left(\Gamma_{f p}\right)}\left(R_{E, p f, 3}\left(\mathbf{U}_{h}^{n}\right),\left(\xi_{p}^{n}-\xi_{p, h}^{n}\right)\right)_{E}, \\
\Upsilon_{12, K}:= & -\sum_{E \in \mathscr{O}_{h}\left(\Gamma_{f p}\right)} \sum_{j=1}^{d-1}\left(R_{E, p f, 4}\left(\mathbf{U}_{h}^{n}\right)(j), \mathbf{v}_{f}^{n}-\mathbf{v}_{f, h}^{n} \cdot \tau_{j}\right)_{E} .
\end{aligned}
$$

Thus, from equations (74) and (78), we obtain

$$
\begin{aligned}
\mathbf{A}_{K}\left(\mathbf{U}^{n}-\mathbf{U}_{h}^{n}, \mathbf{W}^{n}\right):= & \left(\mathbf{R}_{f, K}\left(\mathbf{U}_{h}^{n}\right)-\mathbf{r}_{f, K}\left(\mathbf{U}_{h}^{n}\right), \mathbf{v}_{f}^{n}-\mathbf{v}_{f, h}^{n}\right)_{\Omega_{f} \cap K} \\
& +\left(R_{f, K}\left(\mathbf{U}_{h}^{n}\right)-r_{f, K}\left(\mathbf{U}_{h}^{n}\right), w_{f}^{n}-w_{f, h}^{n}\right)_{\Omega_{f} \cap K} \\
& +\left(\mathbf{R}_{p, K, 1}\left(\mathbf{U}_{h}^{n}\right)-\mathbf{r}_{p, K, 1}\left(\mathbf{U}_{h}^{n}\right), \xi_{p}^{n}-\xi_{p, h}^{n}\right)_{\Omega_{p} \cap K} \\
& +\left(R_{p, K}\left(\mathbf{U}_{h}^{n}\right)-r_{p, K}\left(\mathbf{U}_{h}^{n}\right), w_{p}^{n}-w_{p, h}^{n}\right)_{\Omega_{p} \cap K} \\
& +\sum_{i=1}^{12} \eta_{i, K} .
\end{aligned}
$$

Coercivity of operator A leads to inf-sup condition as follows:

$$
\left\|\mathbf{U}^{n}-\mathbf{U}_{h}^{n}\right\|_{\mathbf{H}_{h}} \leq \sup _{\mathbf{W}^{n} \in \mathbf{H}} \frac{\left|\mathbf{A}\left(\mathbf{U}^{n}-\mathbf{U}_{h}^{n}, \mathbf{W}^{n}\right)\right|}{\left\|\mathbf{W}^{n}\right\|_{\mathbf{H}}} .
$$

By consequently, identities (73) and (80), inf-sup condition of operator A (81), Cauchy-Schwarz inequality, estimation of Lemma 4, and the approximation properties of Lemma 3 imply the required estimate and finish the proof.

3.5. Efficiency of the A Posteriori Error Estimator. For $K \in \mathscr{T}_{h}$, we set

$$
\begin{aligned}
\mathbf{H}_{h}(K)= & \mathbf{V}_{f, h}(K) \times W_{f, h}(K) \times \mathbf{V}_{p, h}(K) \\
& \times W_{p, h}(K) \times \mathbf{X}_{p, h}(K) \times \Lambda_{h}(K),
\end{aligned}
$$

where

$$
\begin{aligned}
\mathbf{V}_{f, h}(K) & :=\left\{\mathbf{v}_{f, h \mid K}: \mathbf{v}_{f, h} \in \mathbf{V}_{f, h}\right\}, \\
W_{f, h}(K) & :=\left\{w_{f, h \mid K}: w_{f, h} \in W_{f, h}\right\} ; \\
\mathbf{V}_{p, h}(K) & :=\left\{\mathbf{v}_{p, h \mid K}: \mathbf{v}_{p, h} \in \mathbf{V}_{p, h}\right\}, \\
W_{p, h}(K) & :=\left\{w_{p, h \mid K}: w_{p, h} \in W_{p, h}\right\} ; \\
\mathbf{X}_{p, h}(K) & :=\left\{\xi_{p, h \mid K}: \xi_{p, h} \in \mathbf{X}_{p, h}\right\}, \\
\Lambda_{h}(K) & :=\left\{\lambda_{h \mid K}: \lambda_{h} \in \Lambda_{h}\right\} .
\end{aligned}
$$


The family estimator $\left(\Theta_{K}\right)_{K \in \mathscr{T}_{h}}$ is consider efficient if it satisfies the following theorem.

Theorem 4 (lower error bound). Let $\mathbf{U}=\left(\mathbf{U}^{n}\right)_{n=0}^{N}$ such that $\mathbf{U}^{n}=\left(\mathbf{u}_{f}^{n}, p_{f}^{n}, \mathbf{u}_{p}^{n}, p_{p}^{n}, \eta_{p}^{n}, \lambda^{n}\right) \in \mathbf{H}$ be the exact solution and $\mathbf{U}_{h}=\left(\mathbf{U}_{h}^{n}\right)_{n=0}^{N}$ with $\mathbf{U}_{h}^{n}=\left(\mathbf{u}_{f, h}^{n}, p_{f, h}^{n}, \mathbf{u}_{p, h}^{n}, p_{p, h}^{n}, \eta_{p, h}^{n}, \lambda_{h}^{n}\right) \in \mathbf{H}_{h}$ be the finite element solution. Then, there exist a positive constant $C_{\text {low }}$ such that the error is bounded locally from below for all $K \in \mathscr{T}_{h}$ by

$$
\Theta_{K} \leq C_{\text {low }}\left[\left\|\mathbf{U}-\mathbf{U}_{h}\right\|_{l^{\infty}\left(0, T ; \mathbf{H}_{h}(K)\right)}+\sum_{K^{\prime} \subset \widetilde{w}_{K}} \zeta_{K^{\prime}}\right],
$$

where $\widetilde{w}_{K}$ is a finite union of neighbouring elements of $K$ and $\|\cdot\|_{\mathbf{H}_{h}(K)}$ is the product norm.

Proof. The lower bound is proved using the standard elementwise integration by parts, namely, error equation of Section 3.3 (i.e., identity (73) and equation (80)) and some inverse estimates of Lemma 1 (cf. [38] for details).

\section{Discussion}

In this paper, we have discussed a posteriori error estimates for a finite element approximation of the Stokes-Biot system where homogeneous boundary conditions are employed. The approach utilizes a fully discrete conforming finite element method. A residual type a posteriori error estimator is provided, that is, both reliable and efficient.

In a future paper, we will study the influence of nonhomogeneous boundary conditions on the a posteriori error indicators presented in this work. Further, it is well known that an internal layer appears at the interface $\Gamma_{f p}$ as the permeability tensor degenerates; in that case, anisotropic meshes have to be used in this layer (see, for instance, [10]). Hence, we intend to extend our results to such anisotropic meshes.

\section{Nomenclatures}

(i) $\Omega \subset \mathbb{R}^{d}, d \in\{2,3\}$ : bounded domain

(ii) $\Omega_{p}$ : the poroelastic medium domain

(iii) $\Omega_{f}=\Omega \backslash \bar{\Omega}_{d}$

(iv) $\Gamma_{f p}=\partial \Omega_{f} \cap \partial \Omega_{p}$

(v) $\Gamma_{*}=\partial \Omega_{*} \backslash \Gamma_{f p}, *=f, p$

(vi) $\mathbf{n}_{f}$ (respectively, $\mathbf{n}_{p}$ ): the unit outward normal vector along $\partial \Omega_{f}$ (respectively, $\partial \Omega_{p}$ )

(vii) $\mathbf{u}_{f}$ : the fluid velocity in $\Omega_{f}$

(viii) $p_{f}$ : the fluid pressure in $\Omega_{f}$

(ix) $\mathbf{u}_{p}, \eta_{p}$ : the fluid velocities in $\Omega_{p}$

(x) $p_{p}$ : the fluid pressure in $\Omega_{p}$

(xi) In $2 D$, the curl of a scalar function $w$ is given as usual by $\operatorname{curl} w:=\left(\left(\partial w / \partial x_{2}\right),-\left(\partial w / \partial x_{1}\right)\right)^{\top}$

(xii) In $3 D$, the curl of a vector function $\mathbf{w}=\left(w_{1}\right.$, $\left.w_{2}, w_{3}\right)$ is given as usual by curl $\mathbf{w}:=\nabla \times \mathbf{w}$, namely, curl w := $\left(\left(\partial w_{3} / \partial x_{2}\right)-\left(\partial w_{2} / \partial x_{3}\right), \quad(\partial\right.$ $\left.\left.w_{1} / \partial x_{3}\right)-\left(\partial w_{3} / \partial x_{1}\right),\left(\partial w_{2} / \partial x_{1}\right)-\left(\partial w_{1} / \partial x_{2}\right)\right)$

(xiii) $\mathbb{P}^{k}$ : the space of polynomials of total degree not larger than $k$

(xiv) $\mathscr{T}_{h}$ : triangulation of $\Omega$

(xv) $\mathscr{T}_{h}^{*}$ : the corresponding induced triangulation of $\Omega_{*}, * \in\{f, p\}$

(xvi) For any $K \in \mathscr{T}_{h}, h_{K}$ is the diameter of $K$ and $\rho_{K}=2 r_{K}$ is the diameter of the largest ball inscribed into $K$

(xvii) $h:=\max _{K \in \mathscr{T}_{h}} h_{K}$ and $\sigma_{h}:=\max _{K \in \mathscr{T}_{h}}\left(h_{K} / \rho_{K}\right)$

(xviii) $\mathscr{E}_{h}$ : the set of all the edges or faces of the triangulation

(xix) $\mathscr{E}(K)$ : the set of all the edges $(N=2)$ or faces $(N=3)$ of a element $K$

(xx) $\mathscr{E}_{h}:=\cup_{K \in \mathscr{T}_{h}} \mathscr{E}(K)$

(xxi) $\mathcal{N}(K)$ : the set of all the vertices of a element $K$

(xxii) $\mathcal{N}_{h}:=\cup_{K \in \mathscr{T}_{h}} \mathcal{N}(K)$

(xxiii) For $\mathscr{A} \subset \bar{\Omega}, \mathscr{E}_{h}(\mathscr{A}):=\left\{E \in \mathscr{E}_{h}: E \subset \mathscr{A}\right\}$

(xxiv) For $E \in \mathscr{E}_{h}\left(\Omega_{*}\right)$, we associate a unit vector $\mathbf{n}_{E, *}$ such that $\mathbf{n}_{E, *}$ is orthogonal to $E$ and equals to the unit exterior normal vector to $\partial \Omega_{*}$, $* \in\{f, p\}$

(xxv) For $E \in \mathscr{E}_{h}\left(\Omega_{*}\right),[\phi]_{E}$ is the jump across $E$ in the direction of $\mathbf{n}_{E, *}$

(xxvi) In order to avoid excessive use of constants, the abbreviations $x \leqq y$ and $x \sim y$ stand for $x \leq c y$ and $c_{1} x \leq y \leq c_{2} x$, respectively, with positive constants independent of $x, y$ or $\mathscr{T}_{h}$

(xxvii) $\partial_{t}=\left(\partial / \partial_{t}\right)$

(xxviii) $\|\phi\|_{L^{2}(0, T ; X)}:=\left[\int_{0}^{T}\|\phi(s)\|_{X}^{2} \mathrm{~d} s\right]^{1 / 2}$

(xxix) $\|\phi\|_{L^{\infty}(0, T ; X)}:=\operatorname{esssup}_{0 \leq t \leq T}\|\phi(t)\|_{X}$

$(\mathrm{xxx})\|\phi\|_{W^{1, \infty}(0, T ; X)}:=\operatorname{esssup}_{0 \leq t \leq T}\left\{\|\phi(t)\|_{X},\left\|\partial_{t} \phi(t)\right\|_{X}\right\}$

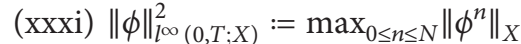

$\left(\right.$ xxxii) $\|\phi\|_{l^{2}(0, T ; X)}:=\left(\tau \sum_{n=0}^{N}\left\|\phi^{n}\right\|_{X}^{2}\right)^{1 / 2}$

(xxxiii) $\|\cdot\|_{\mathbf{H}_{h}}$ : product norm on $\mathbf{H}_{h}$

(xxxiv) $\|\cdot\|_{\mathbf{H}_{h}(K)}$ : local product norm on $\mathbf{H}_{h}(K)$.

\section{Data Availability}

There are no data underlying the findings in this paper to be shared.

\section{Disclosure}

The results presented in this paper constitute a continuation of our work posted on arxiv at the following link: https:// arxiv.org/abs/2004.10676.

\section{Conflicts of Interest}

The authors declare that they have no conflicts of interest. 


\section{References}

[1] M. A. Biot, "General theory of three-dimensional consolidation," Journal of Applied Physics, vol. 12, no. 2, pp. 155-164, 1941.

[2] B. Ahounou, W. Houédanou, and S. Nicaise, "A residualbased posteriori error estimates for a nonconforming finite element discretization of the Stokes-Darcy coupled problem: isotropic discretization," Afrika Matematika, African Mathematical Union and Springer-Verlag Berlin Heidelberg, vol. 27, no. 3, pp. 701-729, 2016.

[3] S. Caucao, G. N. Gatica, and R. Oyarzùa, "A posteriori error analysis of a fully-mixed formulation for the Navier-Stokes/ Darcy coupled problem with nonlinear viscosity," Computer Methods in Applied Mechanics and Engineering, vol. 315, pp. 943-971, 2016.

[4] S. Caucao, G. N. Gatica, R. Oyarzùa, and I. Šebestovà, "A fullymixed finite element method for the Navier-Stokes/Darcy coupled problem with nonlinear viscosity," Journal of $\mathrm{Nu}$ merical Mathematics, 2015, (in press).

[5] M. Cui and N. Yan, "A posteriori error estimate for the Stokes-Darcy system," Mathematical Methods in the Applied Sciences, vol. 34, no. 9, pp. 1050-1064, 2011.

[6] G. Gatica, R. Oyarzùa, and F.-J. Sayas, "A residual-based a posteriori error estimator for a fully-mixed formulation of the Stokes-Darcy coupled problem," Computer Methods in Applied Mechanics and Engineering, vol. 200, pp. 1877-1891, 2011.

[7] K. W. Houédanou, J. Adetola, and B. Ahounou, "Residualbased a posteriori error estimates for a conforming finite element discretization of the Navier-Stokes/Darcy coupled problem," Journal of Pure and Applied Mathematics: Advances and Applications, vol. 18, no. 1, pp. 37-73, 2017.

[8] K. W. Houédanou and B. Ahounou, "A posteriori error estimation for the Stokes-Darcy coupled problem on anisotropic discretization," Mathematical Methods in the Applied Sciences, vol. 40, no. 10, pp. 3741-3774, 2017.

[9] K. W. Houédanou, "An a posteriori error analysis for a coupled continuum pipe-flow/Darcy model in Karst aquifers: anisotropic and isotropic discretizations," Results in Applied Mathematics, vol. 4, p. 100081, 2019.

[10] K. W. Houédanou, Analyse d'erreur a-posteriori pour quelques méthodes d'éléments finis mixtes pour le problème de transmission Stokes-Darcy: Discrétisations isotrope et anisotrope, Université d'Abomey-Calavi, thèse de Doctorat, Godomey, Benin, 2015, http://hal.archives-ouvertes.fr/tel-01373344.

[11] S. Badia, A. Quaini, and A. Quarteroni, "Coupling Biot and Navier-Stokes equations for modelling fluid-poroelastic media interaction," Journal of Computational Physics, vol. 228, no. 21, pp. 7986-8014, 2009.

[12] S. Deparis, M. Discacciati, G. Fourestey, and A. Quarteroni, "Fluid-structure algorithms based on Steklov-Poincaré operators," Computer Methods in Applied Mechanics and Engineering, vol. 43, no. 1-2, pp. 57-74, 2002.

[13] F. Nobile and C. Vergara, "An effective fluid-structure interaction formulation for vascular dynamics by generalized Robin conditions," SIAM Journal on Scientific Computing, vol. 30, no. 2, pp. 731-763, 2008.

[14] A. Quaini and A. Quarteroni, "A semi-implicit approach for fluid-structure interaction based on an algebraic fractional step method," Mathematical Models and Methods in Applied Sciences, vol. 17, no. 6, pp. 957-985, 2007.

[15] J. Xu and K. Yang, "Well-posedness and robust preconditioners for discretized fluid-structure interaction systems,"
Computer Methods in Applied Mechanics and Engineering, vol. 292, pp. 69-91, 2015.

[16] R. Showalter, "Poroelastic filtration coupled to Stokes flow," in Control Theory of Partial Differntial Equations, Volume 242 of Lect. Notes Pure Appl. Math., pp. 229-241, Chapman \& Hall/ CRC, Boca Raton, FL, USA, 2005.

[17] A. Cesmelioglu, "Analysis of the coupled Navier-Stokes/Biot problem," Journal of Mathematical Analysis and Applications, vol. 456, no. 2, pp. 970-991, 2017.

[18] R. Lan, M. J. Ramirez, and P. Sun, "Finite element analysis of an arbitrary Lagrangian-Eulerian method for Stokes/parabolic moving interface problem with jump coefficients," Results in Applied Mathematics, vol. 8, Article ID 100091, 2020.

[19] A. Cesmelioglu and P. Chidyagwai, "Numerical analysis of the coupling of free fluid with a poroelastic material," Numerical Methods for Partial Differential Equations, vol. 36, no. 3, pp. 463-494, 2020.

[20] J. Wen and Y. He, "A strongly conservative finite element method for the coupled Stokes-Biot Model," Computers \& Mathematics with Applications, vol. 80, no. 5, pp. 1421-1442, 2020.

[21] E. A. Bergkamp, C. V. Verhoosel, J. J. C. Remmers, and D. M. J. Smeulders, "A staggered finite element procedure for the coupled Stokes-Biot system with fluid entry resistance," Computational Geosciences, vol. 24, no. 4, pp. 1497-1522, 2020 .

[22] I. Ambartsumyan, E. Khattatov, T. Nguyen, and I. Yotov, "Flow and transport in fractured poroelastic media," GEM International Journal on Geomathematics, vol. 10, no. 1, p. 11, 2019.

[23] I. Ambartsumyan, E. Khattatov, I. Yotov, and P. Zunino, “A Lagrange multiplier method for a Stokes-Biot fluid-poroelastic structure interaction model," Numerische Mathematik, vol. 140, no. 2, pp. 513-553, 2018.

[24] I. Babuška and W. Rheinboldt, "Error estimates for adaptive finite element method," International Journal for Numerical Methods in Engineering, vol. 10, 1978.

[25] I. Babuška and R. Rodriguez, "The problem of the selection of an a-posteriori error indicator based on smoothing techniques," International Journal for Numerical Methods in Engineering, vol. 36, pp. 539-567, 1993.

[26] I. Babuška and W. C. Rheinboldt, "Error estimates for adaptative finite element computations," SIAM Journal on Numerical Analysis, vol. 15, pp. 736-754, 1978.

[27] I. Babuška and W. C. Rheinboldt, "A posteriori error estimates for the finite element method," International Journal for Numerical Methods in Engineering, vol. 12, pp. 1597-1615, 1978.

[28] H. K. Wilfrid, "A posteriori error analysis for a lagrange multiplier method for a stokes/biot fluid-poroelastic structure interaction model," Abstract and Applied Analysis, vol. 2021, Article ID 8877012, 2021.

[29] R. Verfürth, A Review of a Posteriori Error Estimation and Adaptive Mesh-Refinement Techniques, Wiley-Teubner, Chrichester, UK., 1996.

[30] C. Carstensen and G. Dolzmann, "A posteriori error estimates for mixed FEM in elasticity," Numerische Mathematik, vol. 81, no. 2, pp. 187-209, 1998.

[31] G. S. Beavers and D. D. Joseph, "Boundary conditions at a naturally permeable wall," Journal of Fluid Mechanics, vol. 30, no. 1, pp. 197-207, 1967.

[32] R. Adams, Sobolev Spaces, Acadamic Press, INC, Cambridge, MA, USA, 1978. 
[33] J. Galvis and M. Sarkis, "Non-matching motar discretization analysis for the coupled Stokes-Darcy equations," Electronic Transactions on Numerical Analysis, vol. 26, pp. 350-384, 2007.

[34] C. Carstensen, "A posteriori error estimate for the mixed finite element method," Mathematics of Computation, vol. 66, no. 218, pp. 465-476, 1997.

[35] G. Gatica, "A note on the efficiency of residual-based a-posteriori error estimators for some mixed finite element methods," Electronic Transactions on Numerical Analysis, vol. 17, pp. 218-233, 2004.

[36] R. Verfürth, "A posteriori error estimation and adaptive mesh-refinement techniques," Journal of Computational and Applied Mathematics, vol. 50, no. 1-3, pp. 67-83, 1994.

[37] P. Clément, "Approximation by finite element functions using local regularization," Revue française d'automatique, informatique, recherche opérationnelle. Analyse numérique, vol. 9, no. R2, pp. 77-84, 1975.

[38] K. W. Houédanou, J. Adetola, and A. Mohamed, "A posteriori error analysis for a new fully mixed isotropic discretization of the stationary Stokes-Darcy coupled problem," Abstract and Applied Analysis, vol. 2020, Article ID 8628739, 12 pages, 2020. 